\title{
BOUNDARIES OF MARKOV PARTITIONS
}

\author{
JONATHAN ASHLEY, BRUCE KITCHENS AND MATTHEW STAFFORD
}

\begin{abstract}
The core of a Markov partition is the nonwandering set of the map restricted to the boundary of the partition. We show that the core of a Markov partition is always a finitely presented system. Then we show that every one sided sofic system occurs as the core of a Markov partition for an $n$-fold covering map on the circle and every two sided sofic system occurs as the core of a Markov partition for a hyperbolic automorphism of the two dimensional torus.
\end{abstract}

\section{INTRODUCTION}

Markov partitions are often used to produce combinatorial models for dynamical systems. They have been shown to exist in many different settings. It is also known that even in simple seeming settings, the elements of the partition may be geometrically very complicated. One such result says that for a hyperbolic automorphism of $T^{3}$ the intersection of the stable boundaries and the unstable boundaries of the elements of a Markov partition cannot contain a $C^{1}$ arc [Bo3]. We are interested in the dynamics of the boundary. Given a Markov partition for a map, the core of the partition is the nonwandering set of the map restricted to the boundary. We see that the core is always a finitely presented system [F]. Then we concentrate on two simple cases. In the noninvertible case we take the $n$-fold covering maps of the circle. In the invertible case we take the hyperbolic automorphisms of $T^{2}$. In both cases we see that the core must always be a sofic system [W], and that any nonwandering sofic system occurs as the core of some partition.

Section 1 contains a discussion of Markov partitions, their associated subshift of finite type, and the covering map from the subshift of finite type to the dynamical system with the Markov partition.

Section 2 is concerned strictly with symbolic dynamics. There is a discussion of subshifts of finite type, sofic systems, factor maps between symbolic systems and the basic symbolic construction that allows us to realize sofic systems as cores of partitions.

In $\S 3$ we prove that every core is a finitely presented system. Then we restrict our attention to the covering maps of the circle and the automorphisms of $T^{2}$. We present basic examples, show that in both cases the cores must be nonwandering sofic systems and then show that every nonwandering sofic system occurs as the core of a Markov partition in this setting.

Received by the editors April 20, 1990.

1980 Mathematics Subject Classification (1985 Revision). Primary 58F15.

Key words and phrases. Markov partitions, sofic systems. 
In $\S 4$ we discuss the connection between partitions of $S^{1}$ whose elements are intervals and the partitions we have produced.

We would like to thank R. F. Williams. Example 3.5 is his. It was the motivation for this work.

\section{MARKOV PARTITIONS AND THEIR COVERS}

Let $X$ be a compact metric space and $f$ a continuous onto map of $X$ to itself. We say $f$ is positively expansive if there is a $c>0$ so that if $x \neq y$ are points in $X$ there is an $n \in \mathbb{N}$ (we will use $\mathbb{N}=0,1,2, \ldots$ ) so that $d\left(f^{n}(x), f^{n}(y)\right)>c$. When $f$ is invertible, we say $f$ is expansive if there is a $c>0$ so that if $x \neq y$ are points in $X$ there is an $n \in \mathbb{Z}$ so that $d\left(f^{n}(x), f^{n}(y)\right)>c$. In both cases we refer to $c$ as an expansive constant. When $f$ is a homeomorphism, for $\varepsilon>0$ we define the $\varepsilon$-stable set to be

$$
W_{\varepsilon}^{s}(x)=\left\{y \in X: d\left(f^{n}(x), f^{n}(y)\right) \leq \varepsilon \text { for } n \geq 0\right\}
$$

and the $\varepsilon$-unstable set to be

$$
W_{\varepsilon}^{u}(x)=\left\{y \in X: d^{n}\left(f^{n}(x), f^{n}(y)\right) \leq \varepsilon \text { for } n \leq 0\right\} .
$$

There are two metrization theorems that make dealing with these maps much easier.

Theorem. Let $X$ be a compact metric space and $f$ a continuous onto map from $X$ to itself:

(i) (Coven and Reddy [CR]) if $f$ is positively expansive then there is a metric $d$, compatible with the original metric, an $\varepsilon>0$ and $\lambda>1$ so that when $d(x, y)<\varepsilon, d(f(x), f(y))>\lambda d(x, y)$;

(ii) (Fried [F]) if $f$ is an expansive homeomorphism then there is a metric $d$, compatible with the original metric, an $\varepsilon>0$ and $\lambda>1$ so that when $y \in W_{\varepsilon}^{u}(x), d(f(x), f(y))>\lambda d(x, y)$ when $y \in W_{\varepsilon}^{s}(x)$, $d\left(f^{-1}(x), f^{-1}(y)\right)>\lambda d(x, y)$.

We will always assume that the metrics we use are adapted in this sense. In the case where $f$ is a homeomorphism we say a closed set $R \subseteq X$ is a rectangle if there is an $\varepsilon>0$ so that $\operatorname{diameter}(R)$ is less than $\varepsilon$ and $[x, y]=W_{\varepsilon}^{s}(x) \cap W_{\varepsilon}^{u}(y)$ is a single point in $R$ for all points $x, y$ in $R$. Define $W^{s}(x, R)=W_{\varepsilon}^{s}(x) \cap R$ and $W^{u}(x, R)=W_{\varepsilon}^{u}(x) \cap R$. For each $x \in R$ there is a homeomorphism between $R$ and $W^{s}(x, R) \times W^{u}(x, R)$. Say $R$ is proper if $\operatorname{cl}($ int $R)=R$. For a proper rectangle define the stable boundary, $\partial^{s} R$, to be $\left\{x \in R: W^{s}(x, R) \cap\right.$ int $\left.R=\varnothing\right\}$ and the unstable boundary, $\partial^{u} R$, to be $\left\{x \in R: W^{u}(x, R) \cap\right.$ int $\left.R=\varnothing\right\}$. It follows that $\partial R=\partial^{s} R \cup \partial^{u} R$. The familiar picture of a rectangle is a rectangle in the plane where the vertical sides are the stable boundary and the horizontal sides are the unstable boundary. Given $x \in R$, and $\varepsilon$ in the definition of $R$ we may define $\partial W^{s}(x, R)$ and int $W^{s}(x, R)$ to be the boundary and the interior of $W^{s}(x, R)$ as subsets of $W_{\varepsilon}^{s}(x)$. We make analogous definitions for $\partial W^{u}(x, R)$ and int $W^{u}(x, R)$. It follows that $\partial^{s} R \simeq W^{s}(x, R) \times \partial W^{u}(x, R)$ and $\partial^{u} R \simeq \partial^{s} W(x, R) \times W^{u}(x, R)$.

When $f$ is a positively expansive map we say a collection of subset of $X, \mathscr{P}=\left\{R_{1}, \ldots, R_{n}\right\}$ is a Markov partition for $(X, f)$ if: 
(1) $\mathrm{cl}\left(\right.$ int $\left.R_{i}\right)=R_{i}$ for each $R_{i}$,

(2) int $R_{i} \cap$ int $R_{j}=\varnothing$ for $i \neq j$;

(3) the $R_{i}$ 's cover $X$;

(4) if $f\left(\right.$ int $\left.R_{i}\right) \cap$ int $R_{j} \neq \varnothing$ then each point in int $R_{j}$ has one preimage in $R_{i}$.

Condition (4) is the Markov condition.

When $f$ is an expansive homeomorphism we say a collection of subsets of $X, \mathscr{P}=\left\{R_{1}, \ldots, R_{n}\right\}$ is a Markov partition for $(X, f)$ if:

(1) each $R_{i}$ is a proper rectangle;

(2) int $R_{i} \cap$ int $R_{j}=\varnothing$ for $i \neq j$;

(3) the $R_{i}$ 's cover $X$;

(4) for $x \in \operatorname{int} R_{i}, f(x) \in R_{j}$,

$$
f\left(W^{s}\left(x, R_{i}\right)\right) \subseteq W^{s}\left(f(x), R_{j}\right), \quad f^{-1}\left(W^{u}\left(f(x), R_{j}\right)\right) \subseteq W^{u}\left(x, R_{i}\right) .
$$

Condition (4) is the Markov condition, and differs from the one for noninvertible maps. For a more detailed discussion of Markov partitions see [Bol, F, S]. It is common to require the diameter of the elements of the partition to be less than the expansive constant for the map. This is unnecessarily restrictive and would rule out both Examples 1.1 and 1.2, which we want to allow. The seeming pathology that occurs in later examples is not due to this loosening of the definition. It would also occur under the more stringent definition.

Next we will define subshifts of finite type. Let $\{1, \ldots, n\}$ be an $n$-point space with the discrete topology. Form the product spaces $\{1, \ldots, n\}^{\mathbb{N}}$ and $\{1, \ldots, n\}^{\mathbb{Z}}$ with the product topologies. Each has a metric compatible with the topology. Let $d(x, y)=1 / n^{l}$ where $l=\max \left\{t: x_{i}=y_{i}\right.$ for $\left.i \leq t\right\}$ when $x, y \in\{1, \ldots, n\}^{\mathbb{N}}$ and $l=\max \left\{t: x_{i}=y_{i}\right.$ for $\left.|i| \leq t\right\}$ when $x, y \in$ $\{1, \ldots, n\}^{\mathbb{Z}}$. Both of these spaces are Cantor sets. There is a shift transformation, $\sigma$, defined on each by $\sigma(x)_{i}=x_{i+1}$. In each space let

$$
\mathscr{P}=\left\{[1]_{0}, \ldots,[n]_{0}\right\} \quad \text { where }[i]_{0}=\left\{x: x_{0}=i\right\} .
$$

The partitions defined on $\{1, \ldots, n\}^{\mathbb{N}}$ and $\{1, \ldots, n\}^{\mathbb{Z}}$ are both Markov partitions for $\sigma$. If $\left(i_{1}, \ldots, i_{t}\right) \in\{1, \ldots, n\}^{t}$ then the set $\left[i_{1}, \ldots, i_{t}\right]_{r}$ $=\left\{x: x_{r}=i_{1}, x_{r+1}=i_{2}, \ldots, x_{r+t-1}=i_{t}\right\}$ as a subset of $\{1, \ldots, n\}^{\mathbb{N}}$ or $\{1, \ldots, n\}^{\mathbb{Z}}$ is called a cylinder set. In $\{1, \ldots, n\}^{\mathbb{Z}}$, it is a rectangle when $-t+1 \leq r \leq 0$. The dynamical system $\left(\{1, \ldots, n\}^{\mathbb{N}}, \sigma\right)$ is the one sided full $n$-shift. Here, the shift is a continuous, onto, $n$-to-one, positively expansive map. The dynamical system $\left(\{1, \ldots, n\}^{\mathbb{Z}}, \sigma\right)$ is the two sided full $n$-shift. Here, the shift is an expansive homeomorphism. Let $A$ be an $n \times n$ 0-1 matrix. Define $X_{A} \subseteq\{1, \ldots, n\}^{\mathbb{N}}$ by $X_{A}=\left\{x: A_{x_{i} x_{i+1}}=1\right.$ for all $\left.i \in \mathbb{N}\right\}$, and give it the subspace topology. The dynamical system $\left(X_{A}, \sigma\right)$ is the one sided subshift of finite type defined by $A$. Define $\Sigma_{A} \subseteq\{1, \ldots, n\}^{\mathbb{Z}}$ by $\Sigma_{A}=\left\{x: A_{x_{i} x_{i+1}}=1\right.$ for all $i \in \mathbb{Z}\}$, and give it the subspace topology. The dynamical system $\left(\Sigma_{A}, \sigma\right)$ is the two sided subshift of finite type defined by $A$.

Suppose $(X, f)$ is as before and has Markov partition $\mathscr{P}=\left\{R_{1}, \ldots, R_{n}\right\}$. Define a transition matrix $A$ by

$$
A(i, j)= \begin{cases}1 & \text { if } f\left(\operatorname{int}\left(R_{i}\right)\right) \cap \operatorname{int}\left(R_{j}\right) \neq \varnothing, \\ 0 & \text { if } f\left(\operatorname{int}\left(R_{i}\right)\right) \cap \operatorname{int}\left(R_{j}\right)=\varnothing .\end{cases}
$$

When $f$ is noninvertible we say $\mathscr{P}$ is topologically generating if for all $x \in$ $X_{A}, \bigcap_{i \in \mathbb{N}} f^{-i}\left(R_{x_{i}}\right)$ is a single point. When $f$ is a homeomorphism we say $\mathscr{P}$ is topologically generating if for all $x \in \Sigma_{A}, \bigcap_{i \in \mathbb{Z}} f^{-i}\left(R_{x_{i}}\right)$ is a single point. In 
the noninvertible case, when $\mathscr{P}$ is topologically generating there is a continuous, onto, map defined in the obvious way, that makes the following diagram commute.

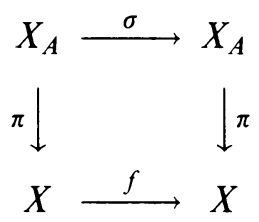

Similarly when $f$ is a homeomorphism we have

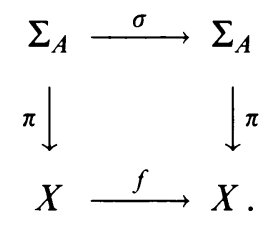

In these cases we say that $\left(X_{A}, \pi\right)$ and $\left(\Sigma_{A}, \pi\right)$ are the covers for $(X, f, \mathscr{P})$. Define the stable boundary of $\mathscr{P}, \partial^{s} \mathscr{P}=\bigcup_{j=1}^{n} \partial^{s} R_{j}$, the unstable boundary of $\mathscr{P}$ similarly, and the boundary of $\mathscr{P}$ to be $\bigcup_{i=1}^{n} \partial R_{i}$. It is easy to see that in the noninvertible case $\pi$ is one-to-one on $X \backslash \bigcup_{i \in \mathbb{N}} f^{-i}(\partial \mathscr{P})$ and in the invertible case $\pi$ is one-to-one on $X \backslash \bigcup_{i \in \mathbb{Z}} f^{-i}(\partial \mathscr{P})$. We will see that $\pi$ is always boundedly finite-to-one. Our main intent is to investigate the properties of $\pi$ and $\partial \mathscr{P}$. We say a Markov partition is metrically generating if for every $x \in X_{A}$ (or $\left.x \in \Sigma_{A}\right), \bigcap_{i \in \mathbb{N}} f^{-i}\left(\right.$ int $R_{X_{i}}$ ) (or $\bigcap_{i \in \mathbb{Z}} f^{-i}$ (int $\left.R_{X_{i}}\right)$ ) is at most a single point. We will see that when $\mathscr{P}$ is metrically generating, there is still a covering map, $\pi$, from $X_{A}$ (or $\Sigma_{A}$ ) to $X$. First, some examples.

Example 1.1. Let $f$ be the doubling map on the circle. If we think of $S^{1}$ as $[0,1]$ with the endpoints identified then $f(x)=2 x(\bmod 1)$. Let $\mathscr{P}=$ $\left\{R_{1}, R_{2}\right\}$ where $R_{1}=[0,1 / 2], R_{2}=[1 / 2,1]$. This partition is metrically but not topologically generating. This is because $\{0,1 / 2\}$ is contained in both $\bigcap_{i \in \mathbb{N}} f^{-i}\left(R_{1}\right)$ and $\bigcap_{i \in \mathbb{N}} f^{-i}\left(R_{2}\right)$. Nevertheless there is a continuous map $\pi:\{1,2\}^{\mathbb{N}} \rightarrow S^{1}$ where the points $.1^{\infty}$ and $.2^{\infty}$ (all 1's and 2's) go to 0 in $S^{1}$ and the points $.12^{\infty}$ and $.21^{\infty}$ go to $1 / 2$ in $S^{1}$. Every other point goes where expected.

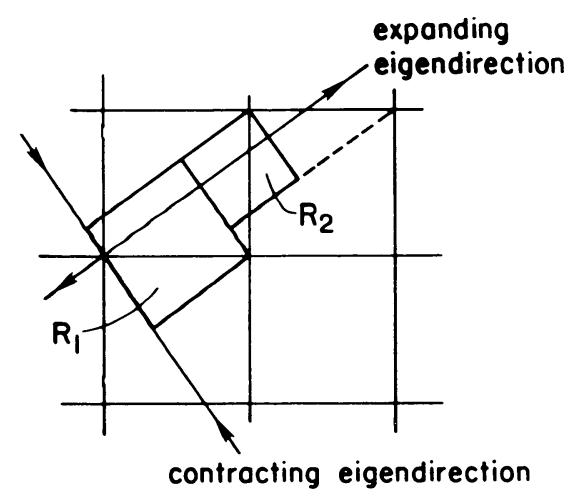

FIGURE 1 
Example 1.2. Let $f$ be the hyperbolic automorphism of the two dimensional torus defined by $\left[\begin{array}{lll}1 & 1 & 1 \\ 1 & 0\end{array}\right][\mathrm{AW}]$. A matrix $A \in G L(n, \mathbb{Z})$ defines an automorphism of $T^{n}$ by letting $A$ act on $\mathbb{R}^{n}$ and then taking the induced automorphism on $T^{n} \simeq \mathbb{R}^{n} / \mathbb{Z}^{n}$. Define a Markov partition on $T^{2}$ by Figure 1 . Here $\mathscr{P}=$ $\left\{R_{1}, R_{2}\right\}$ is topologically generating. The transition matrix is $\left[\begin{array}{ll}1 & 1 \\ 1 & 0\end{array}\right]$ and the covering map $\pi: \Sigma_{A} \rightarrow T^{2}$ is defined as previously described.

Lemma 1.3. Let $\mathscr{P}=\left\{R_{1}, \ldots, R_{n}\right\}$ be a metrically generating partition for $(X, f)$ with transition matrix $A$. Let $\lambda$ be an expansion coefficient for an adapted metric $d$. Then there is an $l \in \mathbb{N}$ so that:

(i) when $f$ is noninvertible, diameter $\left(\bigcap_{i \leq t} f^{-i}\left(\right.\right.$ int $\left.\left.R_{X_{i}}\right)\right)<(1 / \lambda)^{t-l}$ for all $t>l$ and $x \in X_{A}$;

(ii) when $f$ is invertible, diameter $\left(\bigcap_{i \leq|t|} f^{-i}\left(\right.\right.$ int $\left.\left.R_{x_{i}}\right)\right)<(1 / \lambda)^{t-l}$ for all $t>l$ and $x \in \Sigma_{A}$.

Proof. For $x \in X_{A}, \bigcap_{i \in \mathbb{N}} f^{-i}$ (int $R_{x_{i}}$ ) is at most one point. Since $X_{A}$ is compact, there exists an $l \in \mathbb{N}$ so that for all $x \in X_{A}$ diameter $\left(\bigcap_{i \leq l} f^{-i}\left(\right.\right.$ int $\left.\left.R_{x_{i}}\right)\right)<$ $\delta$ where the $\delta$ gives the neighborhood for local expansiveness in $d$. For any $R_{j}$, diameter(int $R_{j} \cap \bigcap_{i \leq l} f^{-i}\left(\right.$ int $\left.\left.R_{x_{i}}\right)\right)<\delta / \lambda<1 / \lambda$. The assertion follows. The invertible case is similar.

Observation 1.4. Suppose $\mathscr{P}=\left\{R_{1}, \ldots, R_{n}\right\}$ is a metrically generating Markov partition for $(X, f)$ and $A$ is its transition matrix. Then there is a natural covering map $\pi:\left(X_{A}, \sigma\right) \rightarrow(X, f)$ when $f$ is noninvertible or from $\left(\Sigma_{A}, \sigma\right)$ to $(X, f)$ when $f$ is invertible, with $\pi\left([j]_{0}\right)=R_{j}, j=1, \ldots, n$.

Proof. For $x \in X_{A}$ consider $\bigcap_{i \in \mathbb{N}} f^{-i}$ (int $R_{x_{i}}$ ). It is at most a single point. If it is a single point define $\pi$ by $\pi(x)=\bigcap_{i \in \mathbb{N}} f^{-i}$ (int $R_{x_{i}}$ ). As defined the range of $\pi$ is $X \backslash \bigcup_{i \in \mathbb{N}} f^{-i}(\partial \mathscr{P})$. It is a dense $G_{\delta}$ containing all points with a dense orbit (or dense forward and backward orbit). Letting $\partial \pi=\{x \in$ $\left.X_{A}: \bigcap_{i \in \mathbb{N}} f^{-i}\left(R_{x_{i}}\right) \subseteq \partial \mathscr{P}\right\}$ we see that the domain of $\pi$ is $X_{A} \backslash \bigcup_{i \in \mathbb{N}} \sigma^{-i}(\partial \pi)$. It is also a dense $G_{\delta}$. As $\pi$ is defined, $\pi \circ \sigma=\sigma \circ f$. Lemma 1.3 shows that $\pi$ is Hölder continuous so it can be extended to all of $X_{A}$. It is clear that $\pi\left([j]_{0}\right)=R_{j}$ for $j=1, \ldots, n$. A similar argument works for the invertible case.

From now on whenever we speak of a Markov partition $\mathscr{P}$ for $(X, f)$ and its cover $\left(X_{A}, \pi\right)$ or $\left(\Sigma_{A}, \pi\right)$ we mean that $\mathscr{P}$ is metrically generating, $A$ is the transition matrix and $\pi$ is the natural covering map. Notice that when $f$ is not invertible $\pi$ is one-to-one on all points with a dense orbit and when $f$ is invertible $\pi$ is one-to-one on all points with a dense forward and backward orbit.

Two points $x, y \in X_{A}$ (or $\Sigma_{A}$ ) are said to be mutually separated if $x_{i} \neq y_{i}$ for all $i \in \mathbb{N}(i \in \mathbb{Z})$.

Theorem 1.5. Let $\mathscr{P}$ be a Markov partition for $(X, f)$ with transition matrix $A$ :

(i) when $f$ is not invertible $(X, f)$ has cover $\left(X_{A}, \pi\right)$ and $\partial \mathscr{P}=\left\{x \in X: \pi^{-1}(x)\right.$ contains a pair of mutually separated points $\} ;$ 
(ii) when $f$ is invertible $(X, f)$ has cover $\left(\Sigma_{A}, \pi\right)$ and $\bigcap_{i \in \mathbb{Z}} f^{-i}(\partial \mathscr{P})=\left\{x \in X: \pi^{-1}(x)\right.$ contains a pair of mutually separated points $\}$. Proof of (i). First we show that if $R_{j} \subseteq f\left(R_{i}\right)$ then $f: R_{i} \rightarrow f\left(R_{i}\right) \cap R_{j}$ is locally onto. This is immediate if the diameter $R_{i}$ is small because then $f:\left(\right.$ int $\left.R_{i}\right) \rightarrow f\left(\right.$ int $\left.R_{i}\right) \cap$ int $R_{j}$ is a homeomorphism. If diameter of $R_{i}$ is large $f\left(R_{i}\right)$ might "wrap around." By Lemma 1.3 for every $\varepsilon>0$ there is an $N$ so that for $x \in X_{A}$ and $m \geq N$ diameter $\bigcap_{i=0}^{m} f^{-i}\left(\right.$ int $\left.R_{x_{i}}\right)<\varepsilon$. This means $f: \bigcap_{i=0}^{m} f^{-i}\left(\right.$ int $\left.R_{x_{i}}\right) \rightarrow \bigcap_{i=1}^{m} f^{-i}\left(\right.$ int $\left.R_{x_{i}}\right)$ is a homeomorphism. Push forward to see that $f: R_{x_{m-1}} \cap f^{-1}\left(R_{x_{m}}\right) \rightarrow R_{x_{m}}$ is a homeomorphism and we have the desired result.

Next we prove statement (i). Suppose $x \in R_{i} \cap R_{i^{\prime}}$. Then $f(x)$ is also in $\partial \mathscr{P}$. This means there is an $R_{j}$ so that $f(x) \in R_{j}$ and $f\left(R_{i}\right) \supseteq R_{j}$. Since $f$ is onto and locally maps $R_{i}$ onto $R_{j}$ there must be a $R_{j^{\prime}} \neq R_{j}$ so that $f(x) \in R_{j^{\prime}}$ and $f\left(R_{i^{\prime}}\right) \supset R_{j^{\prime}}$. This means $\pi^{-1}(x)$ contains points $y, z$ with $y_{0} \neq z_{0}$ and $y_{1} \neq z_{1}$. Then continue. Conversely, if $\pi^{-1}(x)$ contains two points whose 0 th coordinates disagree, then $x$ is in $\partial \mathscr{P}$.

To prove Theorem 1.5(ii) we need some lemmas. We have $\mathscr{P}$ a Markov partition for $(X, f)$ with $f$ invertible and $\left(\Sigma_{A}, \pi\right)$ its associated cover.

Lemma 1.6. Let $x \in \partial^{s} \mathscr{P}$, and $y, z \in \pi^{-1}(x)$. Suppose int $W^{u}\left(x, R_{y_{0}}\right) \cap$ int $W^{u}\left(x, R_{z_{0}}\right)=\varnothing$. Then $y_{i} \neq z_{i}$ for all $i \geq 0$.

Proof. $f\left(\right.$ int $\left.W^{u}\left(x, R_{y_{0}}\right)\right) \supseteq$ int $W^{u}\left(f(x), R_{y_{1}}\right)$. This means $f\left(\right.$ int $\left.W^{u}\left(x, R_{y_{0}}\right)\right)$ $\cap$ int $W^{u}\left(f(x), R_{z_{1}}\right)=\varnothing$ and $f\left(\right.$ int $\left.R_{y_{0}}\right) \cap$ int $R_{z_{1}}=\varnothing$.

Lemma 1.7. Suppose $y, z \in \pi^{-1}(x)$ with $y_{-1}=z_{-1}$ and $y_{0} \neq z_{0}$, then $y_{i} \neq z_{i}$ for all $i \geq 0$.

Proof. If $f^{-1}(x) \in R_{y_{-1}}, f\left(\right.$ int $\left.R_{y_{-1}}\right) \cap$ int $R_{y_{0}} \neq \varnothing$, and $f\left(\right.$ int $\left.R_{y_{-1}}\right) \cap$ int $R_{z_{0}} \neq$ $\varnothing$ then $x \in \partial^{s} R_{y_{0}} \cap \partial^{s} R_{z_{0}}$ and int $W^{u}\left(x, R_{y_{0}}\right) \cap$ int $W^{u}\left(x, R_{z_{0}}\right)=\varnothing$. Then we can apply Lemma 1.6.

Lemma 1.8. If $y, z \in \pi^{-1}(x)$ and $y_{r}=z_{r}$ and $y_{s}=z_{s}$ for $r<s$ then $y_{i}=z_{i}$ for $r \leq i \leq s$.

Proof. This follows immediately from Lemma 1.7.

Remark 1.9. We say the map has no "diamonds." This was first observed by B. Marcus and gives a simple proof that $\pi$ is boundedly finite-to-one. It means that cardinality of $\pi^{-1}(x)$ is no more than the cardinality of the symbol set of $\Sigma_{A}$ squared. The rest of the proof of Theorem 1.5(ii) is just a combinatorial argument applying the fact that $\pi$ has no diamonds.

Lemma 1.10. Suppose $y, z \in \pi^{-1}(x)$ and $y_{0}=z_{0}$ then $[y, z] \in \pi^{-1}(x)$. Here $[y, z]$ is the point with $[y, z]_{i}=z_{i}$ for $i \leq 0,[y, z]_{i}=y_{i}$ for $i \geq 0$.

Proof. $\pi\left(\left\{w: w_{i}=y_{i}\right.\right.$ for $\left.\left.i \geq 0\right\}\right) \subseteq W^{s}\left(x, R_{y_{0}}\right), \pi\left(\left\{w: w_{i}=z_{i}\right.\right.$ for $i \leq$ $0\}) \subseteq W^{u}\left(x, R_{y_{0}}\right)$. $W^{s}\left(x, R_{y_{0}}\right) \cap W^{u}\left(x, R_{y_{0}}\right)=x$.

Proof of Theorem 1.5(ii). Assume that for each $y, z \in \pi^{-1}(x), y_{i}=z_{i}$ for some $i$. For $y, z \in \pi^{-1}(x)$ assume by Lemma 1.10 that $y_{i}=z_{i}$ for $i \leq 0$ and $y_{i} \neq z_{i}$ for $i>0$. There must be a $w \in \pi^{-1}(x)$ with $w_{0} \neq y_{0}$ and $w_{i} \neq y_{i}$ 
for all $i \leq r$. If this were false then there would be an $r$ so that $w_{i}=y_{i}$ for all $i \leq r$ and all $w \in \pi^{-1}(x)$ because by Remark $1.9 \pi$ is boundedly finite-to-one. Thus $\sigma^{r}(x) \notin \partial \mathscr{P}$. We may find $w \in \pi^{-1}(x)$ with $w_{0} \neq y_{0}, w_{i} \neq y_{i}$ for all $i<r$ and $w_{r}=y_{r}$.

Suppose $r>0$ (see Figure 2). There must be an $r^{\prime}>0$ that $w_{r^{\prime}}=z_{r^{\prime}}$. This forces a diamond. So $r<0$ (see Figure 3).

Let $s<0$ be so that $w_{s}=y_{s}, w_{s+1} \neq y_{s+1}$. There is a $u \in \pi^{-1}(x)$ with $u_{r} \neq y_{r}$. Since $u_{j}=w_{j}, u_{k}=y_{k}$, and $u_{l}=z_{l}$ for some $j, k, l \in \mathbb{Z}$ and the map has no diamonds we must have that $u_{t}=w_{t}=y_{t}=z_{t}$ for some $t$, $r<t \leq s$. (See Figure 4.)

Now the point $v$ defined by $v_{i}=u_{i}$ for $i \leq t, v_{i}=w_{i}$ for $i>t$ is in $\pi^{-1}(x)$ by Lemma 1.10. We have a new point $v$ that satisfies the same conditions as $w$ but $t>r$. Continue and produce a point that is totally separated from $y$. The converse is clear.

If $X$ is a topological space and $g: Y \rightarrow Y$ is a continuous map, a point $x \in Y$ is nonwandering if for every neighborhood $U$ of $x$ there is a $k>0$ so that $f^{k}(U) \cap U \neq \varnothing$. Suppose $\mathscr{P}$ is a Markov partition for $(X, f)$. If $f$ is not invertible then $f$ maps $\partial \mathscr{P}$ into itself and we define the core of $\mathscr{P}, \mathscr{C}(\mathscr{P})$, to be the nonwandering points of $f$ restricted to $\partial \mathscr{P}$. If $f$ is invertible then $\partial \mathscr{P}$ is not invariant under $f$. The intersection, $\bigcap_{i \in \mathbb{Z}} f^{-i}(\partial \mathscr{P})$, is the largest invariant set contained in $\partial \mathscr{P}$. We define the core of $\mathscr{P}, \mathscr{C}(\mathscr{P})$, to be the nonwandering points of $f$ restricted to $\bigcap_{i \in \mathbb{Z}} f^{-i}(\partial \mathscr{P})$.

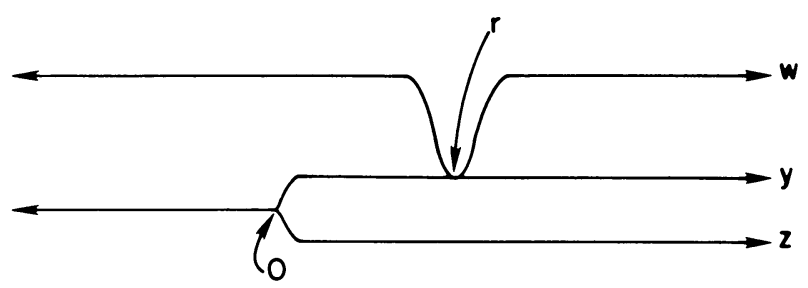

FIGURE 2

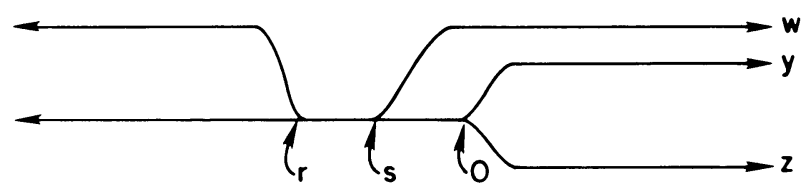

FIGURE 3

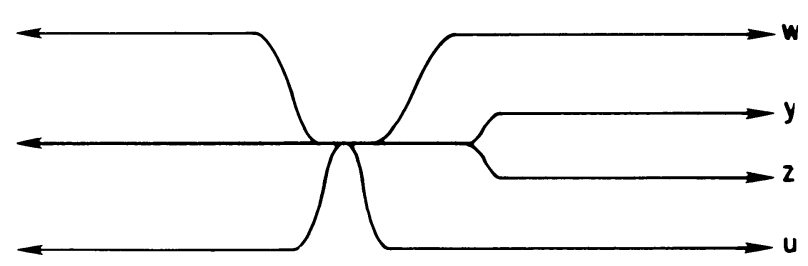

Figure 4 


\section{Symbolic DYNAMicS}

This section is about symbolic dynamics. We will make some definitions and prove some results that we will use in later sections. For a more detailed discussion of ideas about symbolic dynamics see [AM].

In $\S 1$ we defined the one sided $n$-shift $\{1, \ldots, n\}^{\mathbb{N}}$ and the two sided $n$-shift $\{1, \ldots, n\}^{\mathbb{Z}}$ together with their topologies, metrics, and shift transformations. Let $\Lambda$ be a closed shift invariant subset of either fullshift. Put the subspace topology on it and call the dynamical system $(\Lambda, \sigma)$ or $\Lambda$ a subshift. Denote by $\mathscr{W}(\Lambda, n)$ the set of words of length $n$ that occur in $\Lambda,\left\{x_{0} \cdots x_{n-1}: x \in \Lambda\right\}$, and $\mathscr{W}(\Lambda)=\bigcup_{n} \mathscr{W}(\Lambda, n)$ the words that occur in $\Lambda$. For $w \in \mathscr{W}(\Lambda)$ define the follower set of $w, f_{\Lambda}(w)$ or $f(w)$, to be $\{u \in \mathscr{W}(\Lambda): w u \in \mathscr{W}(\Lambda)\}$ and predecessor set, $p_{\Lambda}(w)$ or $p(w)$, to be $\{u \in \mathscr{W}(\Lambda): u w \in \mathscr{W}(S)\}$. We define the $k$ block presentation of $\Lambda$ to be the subshift $\Lambda^{[k]} \subseteq(\mathscr{W}(\Lambda, k))^{\mathbb{N}}$ (or $\left.(\mathscr{W}(\Lambda, k))^{\mathbb{Z}}\right)$ consisting of all points $x=\left(x_{i}\right)=\left(\left(x_{i}^{1} \cdots x_{i}^{k}\right)\right) \in(\mathscr{W}(\Lambda, k))^{\mathbb{N}}$ so that $x_{i}^{2} \cdots x_{i}^{k}=x_{i+1}^{1} \cdots x_{i+1}^{k-1}$ for all $i$ and the point $\left(x_{i}^{1}\right)$ is in $\Lambda$. This is a simple but useful renaming $\left((\Lambda, \sigma)\right.$ and $\left(\Lambda^{[k]}, \sigma\right)$ are topologically conjugate) of $\Lambda$.

There are two types of subshifts that we will deal with. The first type is the subshifts of finite type and the second type is the sofic systems. We will generalize slightly the definition of subshifts of finite type given in $\S 1$. Start with a full $n$-shift, one or two sided, and let $F$ be a finite list of forbidden blocks. Then the subshift of finite type defined by forbidding $F$ is the set $\left\{x \in\{1, \ldots, n\}^{\mathbb{N}}\right.$ : no subblock of $x$ is in $\left.F\right\}$. Suppose $k$ is the length of the longest word in $F$ then consider the $k$ block presentation of $\Lambda$, define transition matrix $A$ by

$$
A\left(i_{1} \cdots i_{k}, j_{1} \cdots j_{k}\right)= \begin{cases}1 & \text { if } i_{r+1}=j_{r}, r=1, \ldots, k-1 \\ 0 & \text { otherwise }\end{cases}
$$

for $i_{1} \cdots i_{k}, j_{1} \cdots j_{k} \in \mathscr{W}(\Lambda, k)$. Then $\Lambda^{[k]}$ is the subshift of finite type $X_{A}$ defined by the transition matrix $A$. This agrees with the definition in §1. A subshift of finite type is a subshift so that for some $k$, the $k$ block presentation is defined by a transition matrix. Equivalently, choose a subset $L \subseteq\{1, \ldots, n\}^{k}$ of allowed blocks and define a subshift of finite type $\Lambda$ to be $\left\{x \in\{1, \ldots, n\}^{\mathbb{N}}: x_{i} \cdots x_{i+k-1} \in L\right.$ all $\left.i\right\}$.

The second type of subshift that interests us is the sofic systems. There are several equivalent definitions [W]. Say $\Lambda$ is a sofic system if there are a finite number of different follower sets, $f_{\Lambda}(w)$, for $w \in \mathscr{W}(\Lambda)$. Or, equivalently, a finite number of predecessor sets $p_{\Lambda}(w)$ for $w \in \mathscr{W}(\Lambda)$. An equivalent definition is that a subshift is sofic if it is the continuous, shift commuting, image of a subshift of finite type. Each sofic system has associated to it two canonical covers $\left(X_{L}, \varphi_{L}\right)$ and $\left(X_{R}, \varphi_{R}\right)$ [Krl]. This means $\varphi_{L}: X_{L} \rightarrow S$ is a continuous onto shift commuting map with some extra properties that will be discussed. A sofic system or a subshift of finite type is irreducible if there is a point with a dense forward orbit. A subshift of finite type is irreducible if and only if its transition matrix is irreducible. A matrix is irreducible if for each pair of vertices $i, j$ there is an $l$ so $\left(A^{l}\right)_{i j}>0$. A sofic system or subshift of finite type is nonwandering if every point in the subshift is a nonwandering point. 


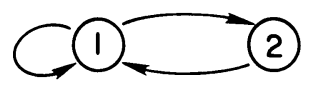

FIGURE 5

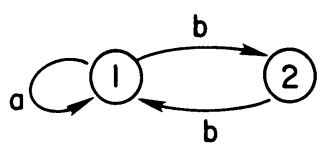

FIGURE 6

A subshift of finite type is nonwandering if and only if there is a permutation of the symbols of the subshift so that the transition matrix is a block diagonal matrix, with each of the square blocks on the diagonal irreducible.

Example 2.1. Let $A=\left[\begin{array}{ll}1 & 1 \\ 1 & 0\end{array}\right]$ the points of $X_{A}\left(\right.$ or $\left.\Sigma_{A}\right)$ are the infinite paths on the graph in Figure 5. We define a map from $X_{A}$ (or $\Sigma_{A}$ ) into $\{a, b\}^{\mathbb{N}}$ (or $\left.\{a, b\}^{\mathbb{Z}}\right)$ by an edge labelling in Figure 6 . This means $\varphi(x)_{i}$ is the label on the edge from $x_{i}$ to $x_{i+1}$. The image under the map is the subshift consisting of all sequence where an even number of $b$ 's occur between two successive $a$ 's. This is a sofic system that is not a shift of finite type. It is usually referred to as the even system.

If $(X, f)$ and $(Y, g)$ are dynamical systems and $\varphi: X \rightarrow Y$ is a continuous onto map so that $\varphi \circ f=g \circ \varphi$ we say that $\varphi$ is a factor map and that $(Y, g)$ is a factor of $(X, f)$. Any continuous shift commuting map between subshifts is a block map [H]. This means there is an $n$ so that $\varphi(x)_{i}=\varphi\left(x_{i} \cdots x_{i+n}\right)$ in the one sided case, and $\varphi(x)_{i}=\varphi\left(x_{i-n}, \ldots, x_{i+n}\right)$ in the two sided case. The map, $\varphi$, depends on a fixed finite number of coordinates. We use $\varphi$ both as a map on points and as a map on blocks. A well-known result [CP] says that a factor map, $\varphi$, between two irreducible sofic systems is either boundedly finite-to-one or some point has uncountably many preimages. For irreducible subshifts of finite type a factor map is finite-to-one if and only if the transition matrices have the same spectral radius [CP]. A factor map $\varphi: S \rightarrow T$ between sofic systems is left-resolving if it is a $k$ block map and whenever $\varphi\left(i_{0} i_{1} \cdots i_{k}\right)=\varphi\left(i_{0}^{\prime} i_{1} \cdots i_{k}\right)$, $i_{0}=i_{0}^{\prime}$. A factor map is right-resolving if it is a $k$ block map and anytime $\varphi\left(i_{0} \cdots i_{k-1} i_{k}\right)=\varphi\left(i_{0} \cdots i_{k-1} i_{k}^{\prime}\right), i_{k}=i_{k}^{\prime}$. Resolving maps are always finite-toone and the left-resolving maps are particularly useful for one-sided shifts.

A finite-to-one factor map between two sided irreducible sofic systems has a degree, $d$. It is the smallest cardinality of the preimages of any point. Every point with a dense forward and backward orbit has exactly $d$ preimages. Say such a map is $d$-to-1 a.e. A left-resolving map between one sided irreducible sofic systems also has a degree, $d$, and every point with a dense orbit has exactly $d$ preimages. A $d$-to-1 a.e. factor map has a magic word. Let $\varphi: S \rightarrow T$ be a $k$ block map between irreducible sofic systems. A word $w=w_{1} \cdots w_{n} \in \mathscr{W}(T)$ is a magic word if there are $d$ blocks $i_{1}^{1} \ldots i_{k}^{1}, i_{1}^{2} \cdots i_{k}^{2}, \ldots, i_{1}^{d} \ldots i_{k}^{d} \in \mathscr{W}(S, k)$ and a $t$ so that every block $u=u_{1} \cdots u_{n+k-1} \in \mathscr{W}(S, n+k-1)$ with $\varphi(u)=w$ has $u_{t} u_{t+1} \cdots u_{t+k-1}=i_{1}^{r} \cdots i_{k}^{r}$ for some $r=1, \ldots, d$. If the map is leftresolving $t=1$, if it is right-resolving $t=n-1$. 
Example 2.2. Examine Example 2.1. The map $\varphi$ is a 2 block 1-to-1 a.e. factor map. It is both left- and right-resolving. The symbol $a$ is a magic word for $\varphi$, only the block 11 maps to it. Such a symbol is a magic symbol.

An irreducible sofic system, as mentioned, has canonical left and right irreducible covers. $\varphi_{L}: X_{L} \rightarrow S$ is a one block, left-resolving one-to-one a.e. cover and $\varphi_{R}: X_{R} \rightarrow S$ is one block, right-resolving (one-to-one a.e. in the two sided case) cover. See $[\mathrm{Kr} 1]$ for more information about these covers.

If $\varphi: X_{A} \rightarrow S$ is a factor map, then $\varphi$ is a $k$ block map for some $k$ and by going to the $k$ block presentation of $X_{A}, X_{A}^{[k]}$, we have a one block factor map $\varphi: X_{A}^{[k]} \rightarrow S$. This is often very useful. For a $d$-to-1 a.e. one block map between irreducible sofic systems, $\varphi: S \rightarrow T$, we say the core of $\varphi, \mathscr{C}(\varphi)$, is the set of points $\left\{y \in T: \varphi^{-1}(y)\right.$ contains $d+1$ mutually separated points $\}$. A collection of points $x^{1}, \ldots, x^{l}$ is mutually separated if $x_{i}^{r} \neq x_{i}^{s}$ for any $1 \leq r \neq s \leq l, i \in \mathbb{N}$ (or $\mathbb{Z}$ ).

Observation 2.3 [F]. If $\varphi: X_{A} \rightarrow X_{B}$ (or $\Sigma_{A} \rightarrow \Sigma_{B}$ ) is a factor map and $\Lambda \subseteq \Sigma_{B}$ then $\Lambda$ is a subshift of finite type if and only if $\varphi^{-1}(\Lambda)$ is a subshift of finite type.

Proof. Suppose $\Lambda$ is a subshift of finite type defined by an allowed list of blocks $L=\left\{b_{1}, \ldots, b_{n}\right\}$. Then $\varphi^{-1}\left(\cup b_{i}\right)$, is open-closed and is a finite union of cylinder sets. $\varphi^{-1}(\Lambda)$ is defined by a finite list of allowed blocks.

Conversely, suppose $\varphi^{-1}(\Lambda)$ is a subshift of finite type and is defined by an allowable list of blocks $\left\{c_{1}, \ldots, c_{m}\right\}$. The complement of $\varphi\left(\bigcup c_{j}\right)$ is closed in $X_{B}$. For sufficiently large $l, \mathscr{W}(\Lambda, l)=\left\{b_{1}, \ldots, b_{n}\right\}$ and $\bigcup b_{j} \subseteq \bigcup c_{i} . \Lambda$ is defined by an allowable list of blocks.

Observation 2.4. If $\varphi: S \rightarrow T$ is a factor map between sofic systems and $\Lambda \subseteq T$, then $\Lambda$ is sofic if and only if $\varphi^{-1}(\Lambda)$ is sofic.

Proof. If $\varphi^{-1}(\Lambda)$ is sofic then $\Lambda$ is sofic by definition.

Suppose $\Lambda$ is sofic. Assume $\varphi$ is a one block map. For a word $w$ that occurs in $\varphi^{-1}(\Lambda) \quad f_{\varphi^{-1}(\Lambda)}(w)=f_{s}(w) \cap \varphi^{-1}\left(f_{\Lambda}(\varphi(w))\right)$. Since there are finitely many distinct follower sets in $S$, and finitely many in $\Lambda$, there are finitely many in $\varphi^{-1}(\Lambda)$.

Observation 2.5. Let $\varphi: X_{A} \rightarrow X_{B}$ (or $\Sigma_{A} \rightarrow \Sigma_{B}$ ) be a finite-to-one map. Then the core of $\varphi, \mathscr{C}(\varphi)$, is a sofic system.

Proof. Go to a higher block presentation of $X_{A}$ so that $\varphi$ is a one block map. Suppose $\varphi$ has degree $d$. Define a new subshift of finite type, $X_{C}$, with symbol set $L_{C}=\left\{\left(i_{1}, \ldots, i_{d+1}\right): i_{r} \in L_{A}, i_{r} \neq i_{s}, r \neq s\right.$, and $\varphi\left(i_{r}\right)=\varphi\left(i_{s}\right)$ all $r, s\}$. Define transitions $\left(i_{1}, \ldots, i_{d+1}\right) \rightarrow\left(j_{1}, \ldots, j_{d+1}\right)$ if $i_{r} \rightarrow j_{r}$ for $r=$ $1, \ldots, d+1$. Define an into block map $\psi: X_{C} \rightarrow X_{B}$ by $\psi\left(\left(i_{1}, \ldots, i_{d+1}\right)\right)=$ $\varphi\left(i_{1}\right)$. Then $\psi\left(X_{C}\right)=\mathscr{C}(\varphi)$ and $\mathscr{C}(\varphi)$ is sofic.

Lemma 2.6. Let $X_{B}\left(\right.$ or $\left.\Sigma_{B}\right)$ be an irreducible subshift of finite type and $X_{C}$ (or $\Sigma_{C}$ ) a subshift of finite type contained in, but not equal to $X_{B}\left(\Sigma_{B}\right)$. Then there is an irreducible subshift of finite type $X_{A}\left(\right.$ or $\left.\Sigma_{A}\right)$ and a one-to-one a.e. left-resolving (or right-resolving) map $\varphi: X_{A} \rightarrow X_{B} \quad\left(\Sigma_{A} \rightarrow \Sigma_{B}\right)$ so that the core of $\varphi$ is $X_{C} \quad\left(\Sigma_{C}\right)$. 
Proof. We first deal with the case where $X_{C}$ is defined by an irreducible subgraph, $G_{C}$, of $G_{B}$ with $G_{B} \backslash G_{C}$ still irreducible (any two vertices of $G_{B} \backslash G_{C}$ are connected by a path) and there are at least two edges beginning in $G_{C}$ and ending outside of $G_{C}$. We will construct the graph $G_{A}$. Let $V_{A}=$ $\left(V_{B} \backslash V_{C}\right) \cup\left(V_{C} \times\{1,2\}\right)$. Define the edges of $G_{A}$ as follows. If $e \in E_{B}$ that begins at $i \notin V_{C}$ and ends at $j \notin V_{C}$ then put an edge in $G_{A}$ beginning at $i$ and ending at $j$. If $e \in E_{B}$ that begins at $i$ and ends at $j \in V_{C}$ put two edges in $G_{A},(e, 1)$ and $(e, 2)$. If $i \notin V_{C}$ then $(e, 1)$ and $(e, 2)$ begin at $i$ and end at $(j, 1)$ and $(j, 2)$, respectively. If $i \in V_{C}$ then $(e, 1)$ begins at $(i, 1)$ and ends at $(j, 1)$ and $(e, 2)$ begins at $(i, 2)$ and ends at $(j, 2)$. Finally consider the edges that begin in $V_{C}$ and end outside of it. Divide these into two nonempty disjoint sets, $E_{1}$ and $E_{2}$. For $e \in E_{1}$ beginning at $i \in V_{C}$ and ending at $j \notin V_{C}$ put an edge in $G_{A}$ that begins at $(i, 1)$ and ends at $j$. Deal with edges in $E_{2}$ is the analogous way. This defines $G_{A}$. The fact that $E_{1}$ and $E_{2}$ are both nonempty means that $G_{A}$ is irreducible. Define a graph homomorphism $\varphi$ from $G_{A}$ to $G_{B}$ by sending each vertex or edge in $G_{A}$ to the vertex or edge in $G_{B}$ that caused it to be produced. Every vertex in $V_{B} \backslash V_{C}$ has one preimage and every vertex in $V_{C}$ has two preimages under $\varphi$. Every edge ending in $V_{B} \backslash V_{C}$ has one preimage and every edge ending in $V_{C}$ has two under $\varphi$. The map defined from $X_{A}$ to $X_{B}$ is left-resolving by construction. If $x \in X_{B}$ and $x_{n} \in V_{B} \backslash V_{C}$ then for $y, z \in \varphi^{-1}(x), y_{i}=z_{i}$ for all $i \leq n$. This means $\varphi$ is one-to-one a.e. and the core of $\varphi$ is $X_{C}$. Example 2.7 illustrates this construction.

There are two problems left, one is that $X_{C}$ may not be irreducible, the other is that $X_{C}$ may not be defined by a subgraph of $G_{B}$ meeting the above requirements.

When $X_{C}$ is not irreducible we need to make sure that each terminal component $G$ of $G_{C}$ has at least two edges going from it to $V_{B} \backslash V_{C}$. A component $G$ of $G_{C}$ is terminal if no edge beginning in $G$ ends at a vertex of $G_{C}$ not in $G$. Then when we form $E_{1}$ and $E_{2}$ we make sure that each terminal component has an edge going from it to $V_{B} \backslash V_{C}$ in $E_{1}$ and in $E_{2}$. This is to insure that $G_{A}$ is irreducible.

When $X_{C}$ is not defined by a subgraph of $G_{B}$ meeting the above requirements we go to a higher block presentation of $X_{B}$ to get $X_{C}$ defined by a subgraph, $G_{C}$, of $G_{B}$ so that $G_{B} \backslash G_{C}$ is irreducible. If $G_{C}$ has two edges beginning in $G_{C}$ and ending in $V_{B} \backslash V_{C}$ we are done. If there is only one such edge we will do some symbol splits (by followers). Suppose the edge is $e$ and it begins at $i \in V_{C}$ and ends at $j \in V_{B} \backslash V_{C}$ and that $f_{B}(j)>1$. Partition $f_{B}(j)$ into two nonempty sets $F_{1}$ and $F_{2}$. Define a new graph $G_{B^{\prime}}$ by leaving $G_{B}$ alone except for $j$ and its incoming and outgoing edges. Replace $j$ by $j_{1}$ and $j_{2}$. Replace each incoming edge $e$ by two edges, $e_{1}$ and $e_{2}$. If $e$ is not a self-loop at $j$ then $e_{1}$ and $e_{2}$ begin where $e$ began, $e_{1}$ ends at $j_{1}$ and $e_{2}$ ends at $j_{2}$. If $e$ is a self-loop then $j$ is in say $F_{1}$. Both $e_{1}$ and $e_{2}$ will begin at $j_{1}$ and end at $j_{1}$ and $j_{2}$, respectively. For each outgoing edge, $e$, (not including a self-loop) ending at $k \in F_{1}$, replace it by a new edge beginning at $j_{1}$ and ending at $k$. Do the analogous thing for edges ending at elements of $F_{2}$. This is an elementary topological conjugacy. $X_{B^{\prime}}$ is topologically conjugate to $X_{B}$, the graph $G_{C}$ is the same in $G_{B}$ and $G_{B^{\prime}}$ but in $G_{B^{\prime}}$ has two outgoing edges. This is an extremely important construction in symbolic dynamics (see [M] for 
further discussion). If the only edge leaving $G_{B}$ ends at a vertex with only one outgoing edge, we will need to do a sequence of these splittings.

Example 2.7. Lemma 2.6 is illustrated by the graphs in Figure 7.

Lemma 2.8. Let $S \subseteq\{1, \ldots, n\}^{\mathbb{N}}$ (or $\{1, \ldots, n\}^{\mathbb{Z}}$ ) be a sofic system, then there is a finite state automaton, $\mathscr{A}_{s}$, with initial state $I$, terminal state $T$, so that the language accepted by $\mathscr{A}_{\bar{s}}, L_{\bar{s}}$, i.e. the labels of paths that begin at $I$ and end at $T$ is $\left\{w_{0} \cdots w_{k} \in\{1, \ldots, n\}^{*}: w_{0} \cdots w_{k-1} \in \mathscr{W}(S)\right.$ but $\left.w_{0} \cdots w_{k} \notin \mathscr{W}(S)\right\}$ (or we may choose $\mathscr{A}_{s}$ so that $L_{\bar{s}}=\left\{w_{0} \cdots w_{k} \in\{1, \ldots, n\}^{*}: w_{1} \cdots w_{k} \in \mathscr{W}(S)\right.$ but $\left.\left.w_{0} \cdots w_{k} \notin \mathscr{W}(S)\right\}\right)$. This means $S=\left\{x:\left[x_{i}, \ldots, x_{i+n}\right] \notin L_{\bar{s}} \forall i, n\right\}$.

Proof. We will make this construction in a right-resolving manner because it is consistent with the usual automata notation and it is easier to follow. For one sided shifts we need left-resolving maps, that is $L_{\bar{s}}=\left\{w: w_{1} \cdots w_{k} \in \mathscr{W}(S)\right.$ but $\left.w_{0} \cdots w_{k} \notin \mathscr{W}(S)\right\}$. To translate from right- to left-resolving we use outgoing instead of incoming edges and read backwards along arrows in the finite state automaton.

Suppose $G_{B}$ is a directed graph and $S$ is obtained by an edge labelling of $G_{B}$. Define the vertices of $\mathscr{A}_{s}$ to be subsets of $V_{B}$, letting $\phi=T$ and taking $I=V_{B}$. For $a \in\{1, \ldots, n\}$ and each subset $U \neq \phi$ of $V_{B}$ define an edge labelled $a$ in $\mathscr{A}_{s}$ from $U$ to $W$, where $W$ is the collection of vertices that can be reached by starting at some vertex in $U$ and following an edge labelled $a$.

The vertex $T=\phi$ has incoming edges but no outgoing ones. This defines the finite state automata $\mathscr{A}_{s}$ that recognizes all words of the form $w_{0} \cdots w_{k}$ where $w_{0} \cdots w_{k-1}$ occurs in $S$ but $w_{0} \cdots w_{k}$ does not. This collection of words is the regular language $L_{\bar{s}}$. Notice that if we take the subshift of finite type defined by $\mathscr{A}_{\bar{s}}$ minus $T$, the edge labelling defines a right-resolving map onto $S$.

Example 2.9. Lemma 2.8 is illustrated by the graphs in Figure 8 .

Theorem 2.10. Let $X_{B}\left(\right.$ or $\left.\Sigma_{B}\right)$ be an irreducible subshift of finite type and $S$ a sofic system contained in but not equal to $X_{B}\left(\right.$ or $\left.\Sigma_{B}\right)$. Then there is an irreducible subshift of finite type $X_{A}\left(\Sigma_{A}\right)$ and a one-to-one a.e. left-resolving (or right) factor map $\varphi: X_{A} \rightarrow X_{B} \quad\left(\Sigma_{A} \rightarrow \Sigma_{B}\right)$ so that the core of $\varphi$ is $S$.
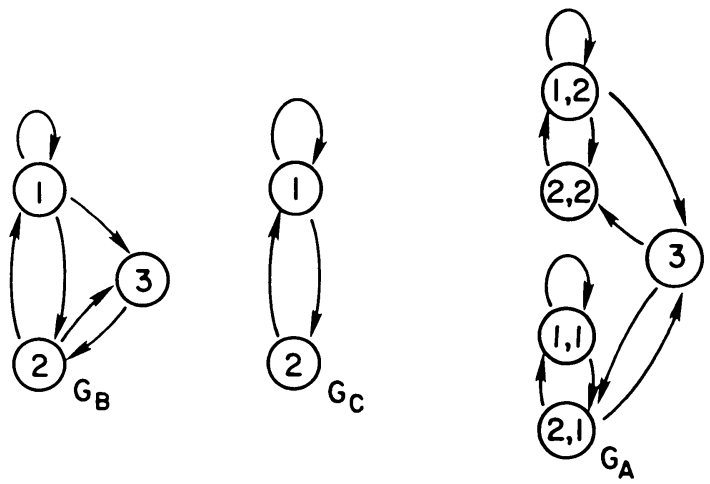

Figure 7 

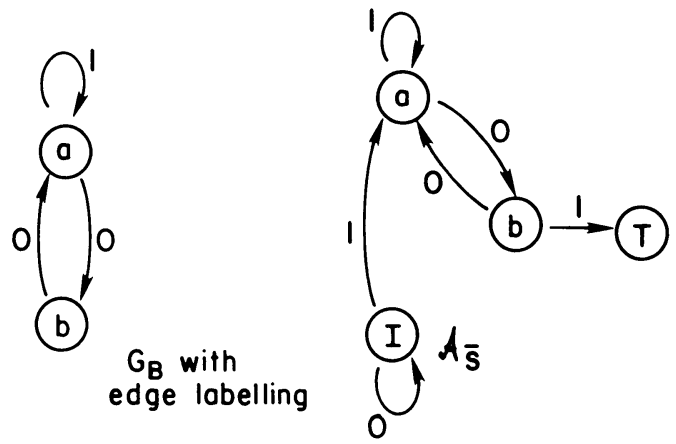

FIGURE 8

Proof. We will use Lemma 2.8 in its left-resolving form, $L_{\bar{s}}=\left\{w_{0} \cdots w_{k}: w_{1} \cdots\right.$ $\left.w_{k} \in \mathscr{W}(S), w_{0} \cdots w_{k} \notin \mathscr{W}(S)\right\}$ to construct a new subshift of finite type $X_{A^{\prime}}$ containing another subshift of finite $X_{C}$ and a one-to-one a.e. left-resolving factor mapping $\varphi^{\prime}: X_{A^{\prime}} \rightarrow X_{B}$ with $\varphi^{\prime}\left(X_{C}\right)=S$ and the core of $\varphi^{\prime}$ contained in $S$. One important point is that, because of Observation 2.3 , when $S$ is not of finite type $\left(\varphi^{\prime}\right)^{-1}(S) \neq X_{C}$. But no periodic point in $S$ will have a preimage outside of $X_{C}$. After this we will apply Lemma 2.7 to $X_{A^{\prime}}$ and $X_{C}$ to get $\psi: X_{A} \rightarrow X_{A^{\prime}}$ with core $X_{C}$ and $\varphi=\varphi^{\prime} \circ \psi$ with core $S$.

Assume we have $X_{B}$ defined by a graph $G_{B}$ with $S \subseteq X_{B}$ and $\mathscr{A}_{s}$ as in Lemma 2.7. Define a new graph, $G$, with labelled edges as follows. The vertices are pairs $(a, W)$ where $a$ is a symbol for $X_{B}$ and $W$ is a subset of the vertices of $\mathscr{A}_{s}$. Say

$$
(b, U) \stackrel{b}{\rightarrow}(a, W)
$$

if $a$ follows $b$ in $X_{B}$ and

$$
U=\left\{i: i \stackrel{b}{\rightarrow} i^{\prime} \text { in } \mathscr{A}_{s} \text {, some } i^{\prime} \in W \text { or } i \stackrel{b}{\rightarrow} I \text { in } \mathscr{A}_{s}\right\} \text {. }
$$

For word $w=w_{0} \cdots w_{k}$, let $w_{0} \cdots w_{k}(I)$ be the vertex in $\mathscr{A}_{\bar{s}}$ (if it exists) that you arrive at by starting at $I$ and following $w$. (Note arrows are followed backwards in $\mathscr{A}_{\bar{s}}$.) If $w \in \mathscr{W}(S)$ then $w_{0} \cdots w_{k}(I)$ is a vertex in $\mathscr{A}_{\bar{s}}$, but not $T$. If $w \in L_{\bar{s}}, w_{0} \cdots w_{k}(I)$ is $T$. If $w$ is in neither it is the empty set. Clearly the edge labelling of $G$ defines a left-resolving factor map from the subshift of finite type defined by $G$ onto $X_{B}$. If $w_{0} \cdots w_{k} \in L_{\bar{s}}$ and $(a, W)$ is a vertex in $G$ with $w_{0} \cdots w_{k}$ leading into it, the path must begin at the state $\left(w_{0}, U_{w}\right)$ where $U_{w}=\left\{\left(w_{0}(I), w_{0} w_{1}(I), \ldots, w_{0} \cdots w_{n-1}(I), T\right\}\right)$ (throw any repeated symbols out of the list). This means every $w_{0} \cdots w_{k} \in L_{\bar{s}}$ is a magic word for the map defined by the edge labelling. So the core of $\varphi^{\prime}$ is contained in $S$. $\varphi^{\prime}$ is left-resolving and one-to-one a.e. There is a unique component of $X_{A^{\prime}}$ of maximal entropy and every other component leads in backward time into it. Call this component $G_{A^{\prime}}$. Let $X_{A^{\prime}}$ be the subshift of finite type defined by $G_{A^{\prime}}$, and $\varphi^{\prime}$ the map onto $X_{B}$ defined by the edge labelling.

Consider the subgraph $G_{C}$ of $G_{A^{\prime}}$ obtained by throwing out any vertex $(a, U)$ where $T \in U$. Let $X_{C}$ be defined by $G_{C}$. Let $x \in S$ be a periodic point and $(a, U)$ be any vertex in $G_{A^{\prime}}$ that occurs in a cycle that maps onto $x$. The subset $U$ cannot contain $T$. This means no periodic point in $S$ has a preimage outside of $X_{C}$ and $\varphi^{\prime}\left(X_{C}\right)=S$. 


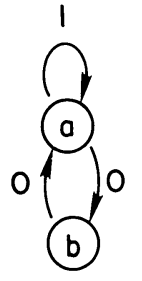

S

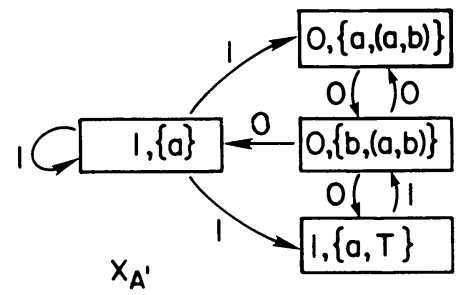

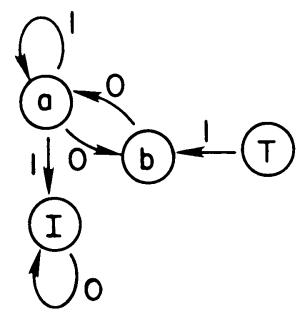

$\mathcal{A}_{\bar{s}}$ moves against the orrows

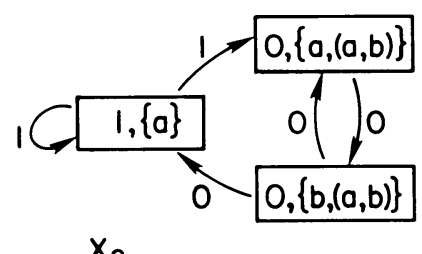

$x_{C}$

FIGURE 9

Finally, we apply Lemma 2.7 to $X_{C} \subseteq X_{A^{\prime}}$ to get $X_{A}$ and $\psi: X_{A} \rightarrow X_{A^{\prime}}$ left-resolving one block map and with the property that every $(a, U)$ where $U$ contains $T$, that is every symbol not in $X_{C}$, has one preimage under $\psi$. We need to see that the core of the composition, $\varphi$, is $S$. By construction $\mathscr{C}(\psi)=X_{C}$ and $\varphi^{\prime}\left(X_{C}\right)=S$. So $\mathscr{C}(\varphi) \supseteq S$. Suppose $w_{0} \cdots w_{k} \in L_{\bar{s}}$. Then every path in $G_{A^{\prime}}$ labelled by $w_{0} \cdots w_{k}$ begins at $\left(w_{0}, U_{w}\right)$ as described earlier. The subset $U_{w}$ contains $T$ so $\left(w_{0}, U_{w}\right)$ has one inverse image under $\varphi^{\prime}$ and so $w_{0} \cdots w_{k}$ is a magic word for $\varphi$. This means $\mathscr{C}(\varphi)=S$.

Notice that constructing a factor map with $S$ as a core is the same as finding a different Markov partition on $X_{B}$ with $S$ as the largest closed invariant set contained in the boundary.

Example 2.11. Theorem 2.10 is illustrated in Figure 9. It is from Example 2.9 but here $\mathscr{A}_{\bar{S}}$ is left-resolving. $S \subseteq\{0,1\}^{\mathbb{N}}=\Sigma_{B}$.

Observation 2.12. Suppose $S$ is an irreducible two sided sofic system $\left(\Sigma_{L}, \varphi_{L}\right)$ and $\left(\Sigma_{R}, \varphi_{R}\right)$ are its irreducible left and right covers. Then the core of the two maps is the same and if $\left(\Sigma_{A}, \varphi\right)$ is any other cover the core of $\varphi_{R}$ is contained in the core of $\varphi$.

Proof. Suppose $x \in S$ and $x$ is not in the core of $\varphi_{R}$. This means there is an $n$ so that $x_{-n} \cdots x_{n} \in \mathscr{W}(S)$ and $f\left(x_{-n} \cdots x_{n}\right)=f\left(w x_{-n} \cdots x_{n}\right)$ for all $w$ such that $w x_{-n} \cdots x_{n} \in \mathscr{W}(S)$. This means $x_{-n} \cdots x_{n}$ is a magic word for $\varphi_{R}$. Consequently, $p\left(x_{-n} \cdots x_{n}\right)=p\left(x_{-n} \cdots x_{n} u\right)$ for all $u$ so that $x_{-n} \cdots x_{n} u \in$ $\mathscr{W}(S)$. The block $x_{-n} \cdots x_{n}$ is also a magic word for $\varphi_{L}$ and $x$ is not in the core of $\varphi_{L}$. By symmetry we see that the two cores are the same. 
Suppose $\left(\Sigma_{A}, \varphi\right)$ is a one block $d$-to-1 a.e. cover for $S$ and $x \in S$ but not in the core of $\varphi$. As before there is an $n$ so that $x_{-n} \cdots x_{n}$ is a magic word for $\varphi$. This means there is a $t,-n \leq t \leq n$, and $i^{1}, \ldots, i^{d}$ so that if $y_{1} \cdots y_{n} \in \mathscr{W}\left(\Sigma_{A}\right)$ with $\varphi\left(y_{1} \cdots y_{n}\right)=x_{1} \cdots x_{n}$ then $y_{t}=i^{r}$ some $1 \leq r \leq d$. Observe that $\varphi\left(f_{A}\left(i^{1}\right)\right)=\cdots=\varphi\left(f_{A}\left(i^{d}\right)\right)$, and $\varphi\left(p_{A}\left(i^{1}\right)\right)=\cdots=\varphi\left(p_{A}\left(i^{d}\right)\right)$. If this were not true $\varphi$ would fail to be $d$-to-1 somewhere. This means that $f_{s}\left(x_{-n} \cdots x_{n}\right)=f\left(u x_{-n} \cdots x_{n}\right)$ for all $u \in \mathscr{W}(S)$ with $u x_{-n} \cdots x_{n} \in \mathscr{W}(S)$. The block $x_{-n} \cdots x_{n}$ is a magic word for $\varphi_{R}$ and $x$ is not in the core of $\varphi_{R}$.

In view of this observation the core of $S$ is well defined. It is defined independently of the presentation of $S$ and is an invariant of topological conjugacy.

\section{SOFIC SYSTEMS AND BOUNDARIES}

An expansive factor of a subshift of finite type is called finitely presented. See [F] for a discussion of the dynamical properties of these systems. A totally disconnected (or 0-dimensional) finitely presented system is a sofic system.

Observation 3.1. Suppose $(X, f)$ is a compact dynamical system and $\mathscr{P}$ is a Markov partition, then:

(i) if $f$ is not invertible $(\partial \mathscr{P}, f)$ is a finitely presented system;

(ii) if $f$ is invertible $\left(\bigcap_{n \in \mathbb{Z}} f^{-n}(\partial \mathscr{P}), f\right)$ is a finitely presented system.

Proof. Let $A$ be the transition matrix for $\mathscr{P}$ and $\left(X_{A}, \pi\right)$ or $\left(\Sigma_{A}, \pi\right)$ be the cover. By Theorem 1.5 we have that

$$
\partial \mathscr{P}=\left\{x: \pi^{-1}(x) \text { contains a pair of mutually separated points }\right\}
$$

in the noninvertible case and

$$
\left(\bigcap_{n \in \mathbb{Z}} f^{-n}(\partial \mathscr{P})\right)=\left\{x: \pi^{-1}(x) \text { contains a pair of mutually separated points }\right\}
$$

in the invertible case. Now make the fibered product of $X_{A}$ or $\Sigma_{A}$ with itself over $X, X_{(A, A)}=\left\{(x, y) \in X_{A} \times X_{A}: \pi(x)=\pi(y)\right\} .\left(X_{(A, A)}, \sigma\right)$ is a subshift of finite type and the projection map $\pi_{1}: X_{(A, A)} \rightarrow X_{A}$ is a one-to-one a.e. factor map. Moreover, $\mathscr{C}\left(\pi_{1}\right)=\pi^{-1}(\partial \mathscr{P})$ and by Observation 2.5 is a sofic system. This means $(\partial \mathscr{P}, f)$ is an expansive image of a subshift of finite type. The same holds in the invertible case.

We will look at two basic examples.

Construction 3.2. The $n$-fold covering map from $S^{1}$ to itself is $f_{n}(x)=n x$ $(\bmod 1)$ where $S^{1}$ is $[0,1]$ with the endpoints identified. Certain Markov partitions are very easy to construct for $f_{n}$. A finite set of points $0 \leq x_{0}<$ $x_{1}<\cdots<x_{k}<1$ partitions the circle into $k+1$ intervals. If this finite set is invariant under $f_{n}$ and the partition metrically generates then we have constructed a Markov partition for $f_{n}$. Any periodic point $z^{0} \in S^{1}$ defines a Markov partition for $f_{n}$ in the following way. Let $f_{n}\left(z^{i}\right)=z^{i+1}$ so the orbit is $z^{0}, \ldots, z^{p-1}$. This set is invariant and if the partition metrically generates we have a Markov partition. If this partition does not generate we add to the collection of points, the points $\left\{y^{0}, \ldots, y^{n-1}\right\}=f_{n}^{-1}\left(z^{0}\right)$. Assume $z^{p-1}=y_{0}$, then the collection of points $\left\{z^{0}, z^{1}, \ldots, z^{p-1}, y^{1}, \ldots, y^{n-1}\right\}$ 
is invariant and the partition it defines metrically generates. Here $\partial \mathscr{P}=$ $\left\{z^{0}, \ldots, z^{p-1}, y^{1}, \ldots, y^{n-1}\right\}$ and $\mathscr{C}(\mathscr{P})=f_{n}(\partial \mathscr{P})=\left\{z^{0}, \ldots, z^{p-1}\right\}$. As an example of this take $z^{0}=0$ the fixed point for $f_{2}$. Then $y^{1}=1 / 2$ and the two points $\{0,1 / 2\}$ define a Markov partition.

Construction 3.3. A hyperbolic automorphism of $T^{2}, f_{\theta}$, is defined by a matrix $\theta \in G L(z, \mathbb{Z})$ with no eigenvalues of modulus one, see Example 1.2. A construction of Bowen [Bo2] allows us to construct some simple Markov partitions for these. The matrix $\theta$ has two real eigenvalues, $\lambda$ with modulus greater than one and $\mu$ with modulus less than one. Let $v_{\lambda}$ and $v_{\mu}$ be eigenvectors for $\lambda$ and $\mu$, respectively, see Figure 10 .

The line, $l_{\lambda}$, passing through the origin containing $v_{\lambda}$ when projected onto $T^{2}$ is $W^{u}(0)$ and the line, $l_{\mu}$, passing through the origin containing $v_{\mu}$ when projected onto the torus is $W^{s}(0)$. Draw fairly long sections of $l_{\lambda}$ and $l_{\mu}$ centered at the origin and project them onto $T^{2}$. Finally extend $l_{\lambda}$ in each direction until each end intersects $l_{\mu}$. Do the same for $l_{\mu}$ to end at intersections with $l_{\lambda}$. These two line segments partition the torus into a finite collection of connected rectangles, $\mathscr{P}$. Since the $\partial^{u} \mathscr{P}$ is a section of $l_{\lambda}$ roughly centered at the origin, $f_{\theta}^{-1}\left(\partial^{u} \mathscr{P}\right) \subseteq \partial^{u} \mathscr{P}$. Similarly for $\partial^{s} \mathscr{P}, f_{\theta}\left(\partial^{s} \mathscr{P}\right) \subseteq \partial^{s} \mathscr{P}$. This guarantees the Markov condition. If the rectangles are small enough, i.e. if $\partial^{u} \mathscr{P}$ and $\partial^{s} \mathscr{P}$ long enough, $\mathscr{P}$ will generate metrically. This produces a Markov partition $\mathscr{P}$. Notice $\bigcap f_{\theta}^{-i}(\partial \mathscr{P})=\mathscr{C}(\mathscr{P})=\{0\}$, a single fixed point. We could equally well make this construction by taking $z^{0} \in T^{2}$ periodic with orbit $z^{0}, \ldots, z^{p-1}$. Make the exact same construction using line segments $l_{\lambda}^{0}, l_{\mu}^{0}$ through $z^{0}$ and parallel to $v_{\lambda}, v_{\mu}$. Then take the partition of $T^{2}$ defined by the line segments $l_{\lambda}^{0}, f_{\theta}\left(l_{\lambda}^{0}\right), \ldots, f_{\theta}^{p-1}\left(l_{\lambda}^{0}\right)$ and $l_{\mu}^{0}, f_{\theta}\left(l_{\mu}^{0}\right), \ldots, f_{\theta}^{p-1}\left(l_{\mu}^{0}\right)$. This will again produce a Markov partition whose boundary is the union of these line segments and with $\bigcap f_{\theta}^{-i}(\partial \mathscr{P})=\mathscr{C}(\mathscr{P})=\left\{z^{0}, \ldots, z^{p-1}\right\}$.

These two constructions produce partitions with finite cores. We make the following observation and then devote the rest of the section to seeing exactly which sofic systems can occur in these settings.

Observation 3.4. (i) Suppose $\mathscr{P}$ is a Markov partition for $f_{n}$ the $n$-fold covering map of $S^{1}$. Then $\left(\partial \mathscr{P}, f_{n}\right)$ and $\left(\mathscr{C}(\mathscr{P}), f_{n}\right)$ are sofic systems.

(ii) Suppose $\mathscr{P}$ is a Markov partition for a hyperbolic toral automorphism $f$ of $T^{2}$. Then $\left(\cap f^{-i}(\mathscr{P}), f\right)$ and $(\mathscr{C}(\mathscr{P}), f)$ are sofic systems.

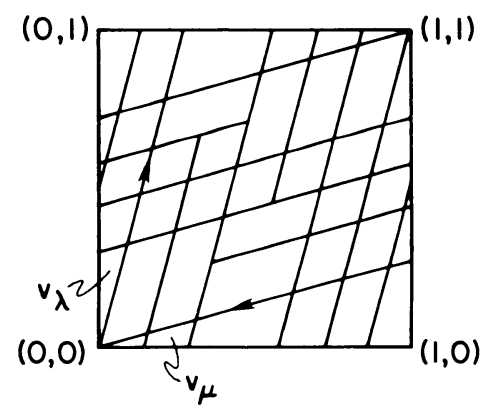

FIGURE 10 


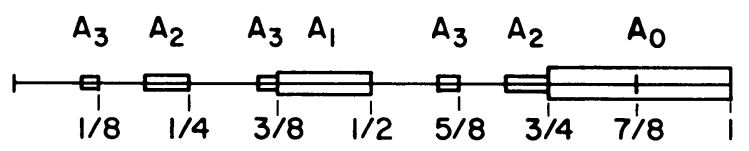

FIGURE 11

Example 3.5. This example is due to R. F. Williams and was the motivation for this work. We want to get the one sided golden mean subshift of finite type as the boundary of a Markov partition for $f_{2}$ on $S^{1}$. It is illustrated by Figure 11 .

Let $A_{0}=[3 / 4,1]$ then $A_{1}=f_{2}^{-1}\left(A_{0}\right) \backslash A_{0}$, and $A_{n}=f_{2}^{-1}\left(A_{n-1}\right) \backslash\left(\bigcup_{i<n} A_{i}\right)$. Then let

$$
\begin{array}{ll}
P_{0}=\mathrm{cl}\left(\left[0, \frac{1}{2}\right] \cap \bigcup_{i \text { even }} A_{i}\right), & P_{2}=\mathrm{cl}\left(\left[\frac{1}{2}, 1\right] \cap \bigcup_{i \text { even }} A_{i}\right), \\
P_{1}=\mathrm{cl}\left(\left[0, \frac{1}{2}\right] \cap \bigcup_{i \text { odd }} A_{i}\right), & P_{3}=\operatorname{cl}\left(\left[\frac{1}{2}, 1\right] \cap \bigcup_{i \text { odd }} A_{i}\right) .
\end{array}
$$

The transition matrix, $A$, for $\mathscr{P}$ is

$$
\left[\begin{array}{llll}
0 & 1 & 0 & 1 \\
1 & 0 & 1 & 0 \\
0 & 1 & 1 & 1 \\
1 & 0 & 0 & 0
\end{array}\right] .
$$

Let $X_{[2]}$ be the full two shift and define $\varphi: X_{A} \rightarrow X_{[2]}$ by a one block map $\varphi(0)=\varphi(2)=0, \varphi(1)=\varphi(3)=1$. Observe that $\varphi$ is left resolving one-toone a.e. and the core of $\varphi$ is the golden mean subshift of finite type. Taking $\pi_{[2]}: X_{[2]} \rightarrow S^{1}$ in the usual way and $\pi=\pi_{[2]} \circ \varphi: X_{A} \rightarrow S^{1}$ we see that $\left(\partial \mathscr{P}, f_{2}\right)=\left(\mathscr{C}(\mathscr{P}), f_{2}\right)$ is topologically conjugate to the golden mean. Intuitively, think of giving points in $S^{1}$ their diadic expansion. Then $A_{0}$ contains all points whose expansion begins with two ones. $A_{1}$ contains all points not in $A_{0}$ that have two ones beginning in the second entry and so forth. The boundary then consists of all points one of whose expansions does not contain a pair of adjacent ones. This is an example of a way to build a partition with a specified boundary. You get a subshift of finite type $X_{A}$ and a factor map $\varphi: X_{A} \rightarrow X_{[2]}$ with the desired core. Make sure the core sits in $X_{[2]}$ so that $\pi_{[2]}: X_{[2]} \rightarrow S^{1}$ will inject the core of $\varphi$ into $S^{1}$. Then the partition is the image of the cylinder sets under $\pi_{[2]} \circ \varphi$ and the boundary is as desired.

Example 3.6. Here we construct the even sofic system of Example 2.1 as the boundary of a Markov partition for $f_{2}$ on $S^{1}$. The construction is similar to Example 3.5. Give the points in the interval their diadic expansion. Let

$$
A_{0}=[.101, .11] \cup[.10001, .1001] \cup \cdots
$$


a countable collection of intervals containing all points whose expansion begins $.10^{n} 1 \cdots$ for odd $n$ (see Figure 12). Let $R_{e}=f_{2}\left(A_{0}\right) \cap[0,1 / 2]$ and $R_{o}=$ $f_{2}\left(A_{0}\right)^{c} \cap[0,1 / 2]$. As before let $A_{n}=f_{2}^{-1}\left(A_{n-1}\right) \backslash \bigcup_{i<n} A_{i}$. Then take

$$
\begin{aligned}
P_{0}=\mathrm{cl}\left(\bigcup_{i \text { even }} A_{i} \cap R_{e}\right), & P_{1}=\mathrm{cl}\left(\bigcup_{i \text { odd }} A_{i} \cap R_{e}\right), \\
P_{2}=\mathrm{cl}\left(\bigcup_{i \text { even }} A_{i} \cap R_{o}\right), & P_{3}=\mathrm{cl}\left(\bigcup_{i \text { odd }} A_{i} \cap R_{o}\right), \\
P_{4}=\operatorname{cl}\left(\bigcup_{i \text { even }} A_{i} \cap\left[\frac{1}{2}, 1\right]\right), & P_{5}=\mathrm{cl}\left(\bigcup_{i \text { odd }} A_{i} \cap\left[\frac{1}{2}, 1\right]\right) .
\end{aligned}
$$

Take $A$ to be the transition matrix, define $\varphi: X_{A} \rightarrow X_{[2]}$ by $\varphi(0)=\varphi(1)=$ $\varphi(2)=\varphi(3)=0, \varphi(4)=\varphi(5)=1$ and observe that the core of $\varphi$ is the even system and that it injects into $S^{1}$ by $\pi_{[2]}$.

Observation 3.7. Suppose $X, Y$ are compact metric spaces, $f$ and $g$ are positively expansive (or expansive) maps of $X$ and $Y$, respectively, onto themselves. Suppose $\mathscr{P}$ is a metrically generating Markov partition for $f$ on $X$ and $\varphi: X \rightarrow Y$ is a factor map that is one-to-one on the transitive (doubly transitive if $f$ is invertible) points of $f$. Then $\varphi(\mathscr{P})$ is a metrically generating Markov partition for $g$ on $Y$.

Proof. We check the conditions needed for a Markov partition. Let $\mathscr{P}=$ $\left\{P_{1}, \ldots, P_{k}\right\}$ and $\mathscr{Q}=\varphi(\mathscr{P})$ with $Q_{i}=\varphi\left(P_{i}\right)$. First, $Q_{i}$ is closed because $P_{i}$ is compact. If $U \subseteq X$ is a nonempty open set the $\varphi(U) \supseteq \varphi\left(U^{c}\right)^{c}$ which is nonempty and open so $\varphi(U)$ has nonempty interior. This means that $\operatorname{cl}\left(\right.$ int $\left.Q_{i}\right)=Q_{i}$. We have int $Q_{i} \cap$ int $Q_{j}=\phi$ for $i \neq j$ otherwise $\varphi$ would not be one-to-one on the points with dense orbits. The $Q_{i}$ cover $X$.

Suppose $f$ and $g$ are not invertible. We need only to see that the Markov condition holds. If $g$ (int $\left.Q_{i}\right) \cap$ int $Q_{j}$ and $y \in$ int $Q_{i}$ then there is a unique $x \in Q_{i}$ with $g(x)=y$. There must be at least one $x$ since $P_{i}$ satisfies this condition in $X$. Suppose there is a $y \in \operatorname{int}\left(Q_{j}\right)$ and $x^{1}, x^{2} \in Q_{i}$ with $g\left(x^{1}\right)=g\left(x^{2}\right)=y$. Then there is a $z$ close to $y$ but with a dense orbit that has this property. That is clearly impossible.

Suppose $f$ and $g$ are invertible. Observe that since $\varphi$ is a factor map the image of a rectangle must be a rectangle so the $Q_{i}$ 's are rectangles. We need to see that the Markov condition holds. This follows immediately from the fact that it holds in $X$ and $\varphi$ is a factor map. Since $\varphi^{-1}$ (int $\left.Q_{i}\right) \subseteq P_{i}, \mathscr{Q}$ metrically generates.

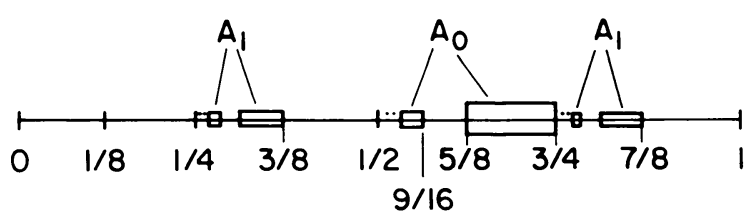

FIGURE 12 
Lemma 3.8. Suppose $X \subseteq S^{1}$ is a closed subset, invariant under $f_{n}$. Further, suppose $\left(X, f_{n}\right)$ is topologically conjugate to a nonwandering sofic system $(S, \sigma)$. Then there is a Markov partition $\mathscr{P}$ for $f_{n}$ so that the core of the boundary is $X$.

Proof. Pick a periodic point $z^{0} \in X$. Use Construction 3.2 to produce a connected Markov partition $\mathscr{Q}$ with standard cover $\left(X_{B}, \pi_{B}\right)$. As noted there, $\partial \mathscr{Q}=\left\{z^{0}, z^{1}, \ldots, z^{p-1}, y^{1}, \ldots, y^{n-1}\right\}$. Consider the subshift $\pi_{B}^{-1}(X)=$ $T \subseteq X_{B}$. This is sofic, by reasoning similar to Observation 2.4 , but is not conjugate to $S$. Each point in $\partial \mathscr{Q}$ has two mutually separated preimages under $\pi_{B}$. Let.$\left(i_{0} i_{1} \cdots i_{p-1}\right)^{\infty}$ and.$\left(i_{0}^{\prime} \cdots i_{p-1}^{\prime}\right)^{\infty}$ be the two points that map to $z^{0}$,.$\left(i_{r} \cdots i_{p-1} i_{0} \cdots i_{r-1}\right)^{\infty}$ and.$\left(i_{r}^{\prime} \cdots i_{p-1}^{\prime} i_{0}^{\prime} \cdots i_{r-1}\right)^{\infty}$ the points that map to $z^{r}, 0 \leq r \leq p-1$, and.$j_{s}\left(i_{0} \cdots i_{p-1}\right)^{\infty}$ and.$j_{s}^{\prime}\left(i_{0}^{\prime} \cdots i_{p-1}^{\prime}\right)^{\infty}$ the points that map to $y^{s}$ for $0 \leq s \leq n-1$. The other points with two preimages under $\pi_{B}$ are points that eventually map to $z^{0}$ by $f_{n}$. Such a point has $\pi_{B}$ preimages.$w j_{s}\left(i_{0} \cdots i_{p-1}\right)^{\infty}$ and.$w j_{s}^{\prime}\left(i_{0}^{\prime} \cdots i_{p-1}\right)^{\infty}$ or.$w\left(i_{r} \cdots i_{p-1} i_{0} \cdots i_{r-1}\right)^{\infty}$ and.$w\left(i_{r} \cdots i_{p-1} i_{0} \cdots i_{r-1}\right)^{\infty}$ for any $w \in \mathscr{W}\left(X_{B}\right)$ so that the preimages are allowable. All other points have single preimages under $\pi_{B}$.

Now apply Theorem 2.10 to $T \subseteq \Sigma_{B}$ to construct $\varphi: X_{A} \rightarrow X_{B}$ left resolving, one-to-one a.e. with $\mathscr{C}(\varphi)=T$. Let $\pi=\pi_{B} \circ \varphi$ and apply Observation 3.5 to $\pi$ to get a Markov partition $\mathscr{P}$ for $f_{n}$. We want to see that the core of $\mathscr{P}$ is $X$. By Theorem $1.5 \partial \mathscr{P}=\left\{x \in S^{\prime}: \pi^{-1}(x)\right.$ contains two mutually separated points $\}$. By construciton $X \subseteq \partial \mathscr{P}$ and since $X$ is nonwandering $X \subseteq \mathscr{C}(\mathscr{P})$. To see the other containment we need to be more careful. The problem could arise from $x \in S^{1}, x \notin \partial \mathscr{Q}$, but $x$ having two preimages under $\pi_{B}$. See Figure 13.

First observe that if $w \notin \mathscr{W}(T)$ then $x$ does not have mutually separated preimages under $\pi$. The problem could be that $w \in \mathscr{W}(T)$ but for some $k, w j_{s}\left(i_{0} \cdots i_{p-1}\right)^{k}, w j_{s}^{\prime}\left(i_{0}^{\prime} \cdots i_{p}^{\prime}\right)^{k} \notin \mathscr{W}(T)$. Then $x$ could possibly have mutually separated preimages under $\pi$. Such a point $x \in \partial \mathscr{P}$ is wandering in $\partial \mathscr{P}$. There are only countably many such points. They accumulate only on points in $X$, and are eventually mapped onto $z^{0}$ by $f_{n}$. It means that $\partial \mathscr{P}$ may be larger than $X \cup\left\{y^{1}, \ldots, y^{n-1}\right\}$, but nevertheless $\mathscr{C}(\mathscr{P})=X$.

Lemma 3.9. Suppose $X \subseteq T^{2}$ is a closed subset, invariant under a hyperbolic automorphism $f_{\theta}$. Further suppose $\left(X, f_{\theta}\right)$ is topologically conjugate to a nonwandering sofic system $(S, \sigma)$. Then there is a Markov partition $\mathscr{P}$ for $f$ so that the core of the partition is $X$.

Proof. The proof of this is very similar to the proof of Lemma 3.8. Pick a periodic point $z^{0} \in X$ and use Construction 3.3 to get a connected Markov partition $\mathscr{Q}$ with standard cover $\left(\Sigma_{B}, \pi_{B}\right)$.

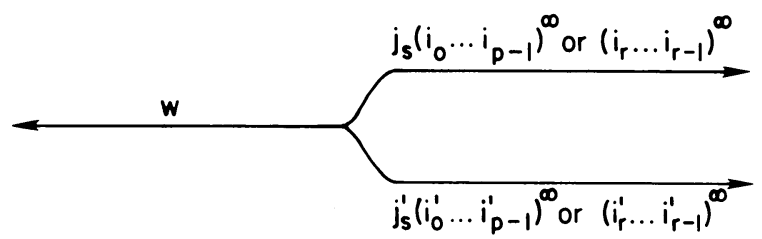

FIGURE 13 
(i)

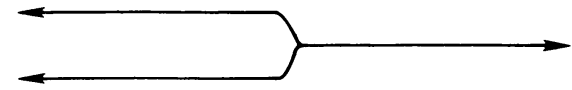

(ii)

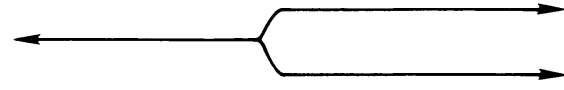

(iii)

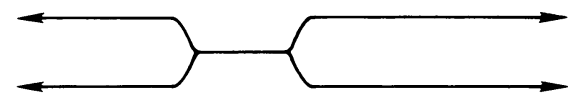

FIGURE 14

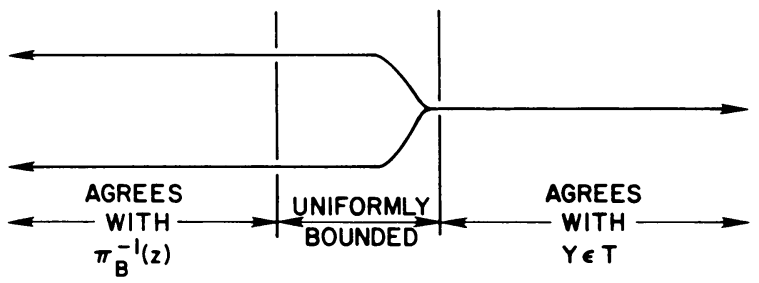

FIGURE 15

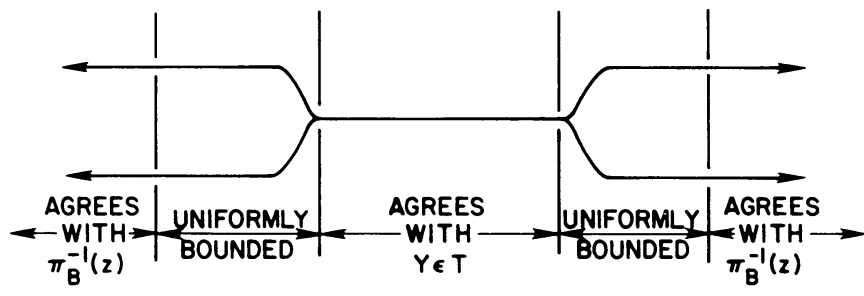

FIGURE 16

As in the previous proof, let $T=\pi_{B}^{-1}(X) \subseteq \Sigma_{B}$. Apply Theorem 2.10 to $T \subseteq \Sigma_{B}$ to construct $\varphi: \Sigma_{A} \rightarrow \Sigma_{B}$ left-resolving, one-to-one a.e. with $\mathscr{C}(\varphi)=$ $T$. Let $\pi=\pi_{B} \circ \varphi$ and $\mathscr{P}$ be the Markov partition defined by Observation 3.5. We want to see the core is $X$. Again by Theorem 1.5 we see that $X$ is in the core of $\mathscr{P}$. The problem is again the other containment. We must examine how points not in $X$ may have mutually separated preimages. Such a point $x \in T^{2}$ must have more than one preimage under $\pi_{B}$. These preimages cannot be mutually separated. They could be one of three types which we represent schematically in Figure 14.

We will examine each type in turn. In the first type there are $\pi_{B}$ preimages as represented but mutually separated $\pi$ preimages. If any word $y_{k} \cdots y_{k+l} \notin$ $\mathscr{W}(T)$ then the $\pi$ preimages are not mutually separated. We have Figure 15.

Considered as points inside $\pi_{B}^{-1}\left(\cap f^{-i}(\partial \mathscr{P})\right) \subseteq \Sigma_{B}$ they are wandering and accumulate only on $T$. In case (ii) the reasoning is exactly the same. In case (iii) we see that the following holds. (See Figure 16.) The same reasoning holds and we see that the nonwandering set of $\pi_{B}^{-1}\left(\cap f^{-i}(\partial \mathscr{P})\right)$ is contained in $T$ and $X$ is the core of $\mathscr{P}$.

Now we prove the main theorem of this section. Notice that the statement in the invertible case is stronger than in the noninvertible case. This is because 
in the invertible (two sided) case we have Krieger's embedding theorem [Kr2]: A sofic system $S$ is topologically conjugate to a proper subshift of a subshift of finite type $\Sigma_{A}$ if and only if $h(S)<h\left(\Sigma_{A}\right)$ and the number of periodic points of each period in $S$ does not exceed the number for the same period in $\Sigma_{A}$. In the noninvertible (one sided) case there is no corresponding theorem. We do not have a guess as to what the correct necessary and sufficient conditions are for one subshift of finite type to be conjugate to a proper subshift of another subshift of finite type. This seems to be a difficult question.

Theorem 3.10. (i) $A$ one sided subshift is conjugate to the core of a Markov partition for some $f_{n}$, the $n$-fold covering map of $S^{1}$, if and only if it is a nonwandering sofic system.

(ii) A two sided subshift is conjugate to the core of a Markov partition for $f_{\theta}$, the hyperbolic automorphism of $T^{2}$ defined by $\theta$, if and only if it is a nonwandering sofic system with $h(S)<h\left(f_{\theta}\right)$ and the number of periodic points of each period in $S$ does not exceed the number in $f_{\theta}$.

Proof. (i) The core of a Markov partition must be a nonwandering sofic system. That is Observation 4.1(i). Fix $n$, then $0<1 / n<2 / n<\cdots<n-1 / n<1$ defines a connected Markov partition for $f_{n}$ by Construction 3.2. This partition generates metrically and the full $n$ shift is the cover. The covering map identifies two fixed points, $.1^{\mathbb{N}}$ and.$n^{\mathbb{N}}$, preimages of the fixed point 0 , and no other periodic points. If $S$ is a transitive sofic system, then for all sufficiently large $n, S$ sits inside the full $n$ shift in such a way that it does not contain the fixed points $.1^{\mathbb{N}}$ and.$n^{\mathbb{N}}$. This means the covering map onto $S^{1}$ injects $S$ into $S^{1}$. The map is a homeomorphism since $S$ is compact. Now apply Lemma 3.8 to the image of $S$.

(ii) By Lemma 4.1(ii) the core of a Markov partition must be a nonwandering sofic system. Now fix $\theta \in G L(2, \mathbb{Z})$ and suppose $S$ is a nonwandering sofic system with $h(S)<h\left(f_{\theta}\right)$ and the condition on the periodic points. Since $h(S)<h\left(f_{\theta}\right)$ there will be a $k$ so that the number of points of period $k$ for $S$ is less than the number for $f_{\theta}$. Pick such a $k$ and let $z^{0}$ be a point of period $k$ for $f_{\theta}$ in $T^{2}$. Apply Construction 3.3 to the orbit of $z^{0}$ to construct a Markov partition $\mathscr{P}$ for $f_{\theta}$ on $T^{2}$ with cover $\left(\Sigma_{A}, \sigma\right)$.

We have that $h\left(\Sigma_{A}\right)=h\left(f_{\theta}\right)>h(S)$ and the number of periodic points of each period for $\Sigma_{A}$ and $f_{\theta}$ is the same with the exception of the orbits the map onto the orbit of $z^{0}$. By the choice of $z^{0}, S$ meets the hypotheses of Krieger's embedding theorem for $\Sigma_{A}$ and it can be embedded so that $\pi^{-1}\left(z^{0}\right) \cap S=\varnothing$. This means $\pi$ maps $S$ into $T^{2}$ injectively and since $S$ is compact $\pi$ restricted to $S$ is a homeomorphism. Finally we apply Lemma 3.9 to the image of $S$ in $T^{2}$ to obtain the desired partition.

4

Observation 4.1. A 0-1 matrix is a transition matrix for a Markov partition for $f_{n}$, the $n$-fold covering map on $S^{1}$ if and only if it is aperiodic and has column sum $n$.

Proof. It is easy to see that these conditions are necessary. It follows from a theorem in $[\mathrm{AGW}]$ that these conditions on a transition matrix, $A$, allow 
the construction of a left resolving one-to-one a.e. factor map $\varphi: X_{A} \rightarrow X_{[n]}$, where $X_{[n]}$ is the full $n$-shift. Compose $\varphi$ with the standard covering map $\pi_{[n]}: X_{[n]} \rightarrow S^{1}$ to get a one-to-one a.e. factor map $\pi: X_{A} \rightarrow S^{1}$. Now apply Observation 3.7 to get a partition with $\left(X_{A}, \pi\right)$ as its cover.

Remark 4.2. The transition matrix alone tells nothing about the core of the Markov partition. It is easy to construct a left-resolving one-to-one a.e. factor map $\varphi: X_{[2]} \rightarrow X_{[2]}$ with a nonfinite sofic core. This, in turn, produces a Markov partition for $f_{2}$ on $S^{1}$ with two elements and $\left[\begin{array}{ll}1 & 1 \\ 1 & 1\end{array}\right]$ as a transition matrix with a nonfinite sofic core.

We have produced complicated Markov partitions for $f_{n}$ on $S^{1}$. However, we have produced these by constructing complicated factor maps between subshifts of finite type and then pushing the Markov partition on the domain shift down onto $S^{1}$. In these cases the image shift came from a very simple, connected partition on $S^{1}$. This means that the complicated partition came from chopping up a simple one. We will show that some partitions do not arise in this way, they are inherently complicated.

Let $\mathscr{P}, \mathscr{Q}$ be Markov partitions for $f_{n}$ on $S^{1}$ with covers $\left(X_{A}, \pi_{A}\right)$ and $\left(X_{B}, \pi_{B}\right)$, respectively. We say $\mathscr{P}$ factors through $\mathscr{Q}$ if there is a factor map $\varphi: X_{A} \rightarrow X_{B}$ so that $\pi_{A}=\pi_{B} \circ \varphi$. The examples we have constructed all factor through some connected partition. We will produce a partition that does not factor through any connected partition.

Lemma 4.3. Let $\mathscr{P}, \mathscr{Q}$ be Markov partitions for $f_{n}$ on $S^{1}$, then $\mathscr{P}$ factors through $\mathscr{Q}$ if and only if $\bigvee_{i=0}^{N} f^{-i}(\mathscr{P})$ refines $\mathscr{Q}$ for some $N$.

Proof. A factor map $\varphi: X_{A} \rightarrow X_{B}$ is an $N$ block map for some $N$.

Let $\mathscr{P}=\left\{P_{1}, \ldots, P_{k}\right\}$ with cover $\left(X_{A}, \pi_{A}\right)$ be a metrically generating Markov partition for $f_{n}$ on $S^{1}$. For each $P_{j}$ we will define extreme points $\left(\zeta_{j}, \eta_{j}\right)$, and the interval of $P_{j}$, with the property that $P_{j} \subseteq\left[\zeta_{j}, \eta_{j}\right]$ in a natural way. If the diameter of $P_{j}$ is small it is obvious how to do this. Choose the $\zeta_{j}$ to the left hand (counter clockwise) endpoint and $\eta_{j}$ to be the right hand (clockwise) endpoint of the smallest closed interval containing $P_{j}$. In general, we know by Lemma 1.3 that there is an $l \in \mathbb{N}$ so that diameter $\left(\bigcap_{i \leq t} f^{-i}\left(P_{x_{i}}\right)\right)<(1 / n)^{t-l}$ for all $t>l$. For large $t$ and $x \in X_{A}$ with $x_{t}=j$ we define the extreme points of $\bigcap_{i \leq t} f^{-i}\left(P_{x_{i}}\right)$ as above. There are $n^{t}$ words, $\left[x_{0}, \ldots, x_{t}\right]$ in $\mathscr{W}\left(X_{A}, t+1\right)$ with $x_{t}=j$. If $x$ and $y$ are two such words there is a rotation of the circle that takes $\bigcap_{i \leq t} f^{-i}\left(P_{x_{i}}\right)$ to $\bigcap_{i \leq t} f^{-i}\left(P_{y_{i}}\right)$. Moreover the smallest closed intervals containing each of them are disjoint. If not, $\mathscr{P}$ would not be metrically generating. This means that diameter $\bigcap_{i \leq t} f^{-i}\left(P_{x_{i}}\right)<(1 / n)^{t}$. For $x, y \in X_{A}$ with $x_{t}=j$ and $f_{n}^{s}(y)=x$, $f^{s}$ takes $\bigcap_{i \leq t+s} f^{-i}\left(P_{y_{i}}\right)$ to $\bigcap_{i \leq t} f^{-i}\left(P_{x_{i}}\right)$ by a homeomorphism. Define the extreme points of $P_{j}$ and the interval of $P_{j}$ to be the image of the extreme points and the image of the interval of $\bigcap_{i \leq t} f^{-i}\left(P_{x_{i}}\right)$, for any large $t$ and $x$ with $x_{t}=j$. Note that the interval of $P_{j}$ is not all of $S^{1}$. We say the collection of extreme points and intervals are the extreme points and intervals for $\mathscr{P}$. The map $f_{n}$ maps extreme points to extreme points and intervals completely over intervals. Each extreme point is either periodic or preperiodic. 
Example 4.4. The partition in Example 3.5 has intervals $\{[0,1 / 3],[0,1 / 2]$, $[1 / 2,2 / 3],[1 / 2,1]\}$.

Lemma 4.5. Suppose $\mathscr{P}$ is a Markov partition for $f_{n}$ on $S^{1}$ and every extreme point of $\mathscr{P}$ is contained in the interval of some $P_{j}$, where it is not an extreme point. Then $\mathscr{P}$ does not factor through any connected partition.

Proof. First observe that if for every $x \in S^{1}$ and every $N \in \mathbb{N}$ there is $y \in X_{A}$ so that $x$ is contained in the interval of $\bigcap_{i \leq N} f_{n}^{-i}\left(P_{y_{i}}\right)$ and is not an extreme point then $\mathscr{P}$ does not factor through any connected partition. This follows because if $Q$ is the connected partition and $x$ is a boundary point then for every $N$ there is a $y \in X_{A}$ so that $\bigcap_{i \leq N} f_{n}^{-i}\left(P_{y_{i}}\right)$ intersects both intervals of $Q$ that are separated by $x$. This means $\bigvee_{i \leq N} f_{n}^{-i}(\mathscr{P})$ never refines $Q$.

Now let $y \in X_{A}$ and $x$ an extreme point of $\bigcap_{i \leq N} f_{n}^{-i}\left(P_{y_{i}}\right)$. By the hypothesis there is a $P_{j}$ whose interval contains $f_{n}^{N}(x)$ and does not have it as an extreme point. This means there is a $z \in X_{A}$ so that $z_{n}=P_{j}, x$ is in the interval of $\bigcap_{i \leq N} f_{n}^{-i}\left(P_{z_{i}}\right)$ and is not an extreme point. Now we apply the observation.

This says that if the extreme points of the partition are interleaved it cannot factor through a connected partition.

Theorem 4.6. A Markov partition $\mathscr{P}$ for $f_{n}$ on $S^{1}$ factors through a connected partition if and only if one of its extreme points is an extreme point for every element of $\mathscr{P}$ whose interval contains it.

Proof. The necessity is proved in Lemma 4.5. To prove sufficiency let this extreme point be $\xi . f_{n}(\xi)$ is an extreme point with the same property, so we may assume $\xi$ is periodic. Apply Construction 3.2 to the orbit of $\xi$ to get a metrically generating connected partition, $Q$. We want to find an $N$ so that $\bigvee_{i \leq N} f^{-i}(\mathscr{P})$ refines $Q$ and then apply Lemma 4.3. Let $X(t)=\{x \in$ $X_{A}: \bigcap_{i<t} f^{-i}\left(P_{x_{i}}\right) \nsubseteq Q_{j}$ any $\left.j\right\}$. This is a closed subset of $X_{A}$ with $X(t+1) \subseteq$ $X(t)$. If $X(N)$ is empty for some $N$ we are done. If no such $N$ exists then by compactness there is a $z \in \bigcap X(t)$. This means $\bigcap_{i \leq t} f^{-i}\left(P_{z_{i}}\right) \nsubseteq Q_{j}$ for any $j$ and all $t$. So, $\pi_{A}(z) \in \partial Q$ and we can assume $\pi_{A}(z)=\xi$. But for each $t, \xi$ is an extreme point for every element of $\bigvee_{i \leq t} f^{-i}(\mathscr{P})$ whose interval contains it. Once the diameter of these sets is small, each set whose interval contains $\xi$ must be contained in one of the two elements of $Q$ separated by $\xi$. A contradiction.

For a detailed discussion of connected partitions see [St].

Example 4.7. This is an example of a Markov partition for $f_{2}$ on $S^{1}$ that does not factor through any connected partition. We see this by finding the extreme points and then applying Theorem 4.6. The construction is similar to that in Examples 3.5 and 3.6. Choose a basic interval $A_{0}=[5 / 8,4 / 9]$ and let

$$
A_{n}=f^{-1}\left(A_{n-1}\right) \backslash \bigcup_{i<n} A_{i} .
$$

We want to see that if $I$ is a preimage interval of $A_{0}, f^{k}(I)=A_{0}$ then either $I \subseteq A_{0}$ or $I \cap A_{0} \subseteq \partial A_{0}$. This is a computation concerning the preimages of $\partial A_{0}$ and the lengths of preimage intervals. 
Define

$$
\begin{array}{rlrl}
P_{0} & =\mathrm{cl}\left(\bigcup_{\text {even }} A_{n} \cap\left[\frac{2}{9}, \frac{5}{9}\right]\right), & P_{1}=\mathrm{cl}\left(\bigcup_{\text {odd }} A_{n} \cap\left[\frac{4}{9}, \frac{7}{9}\right]\right), \\
P_{2}=\mathrm{cl}\left(\bigcup_{\text {even }} A_{n} \cap\left[\frac{5}{9}, \frac{8}{9}\right]\right), & P_{3}=\mathrm{cl}\left(\bigcup_{\text {odd }} A_{n} \cap\left[\frac{7}{9}, \frac{1}{9}\right]\right), \\
P_{4}=\mathrm{cl}\left(\bigcup_{\text {even }} A_{n} \cap\left[\frac{8}{9}, \frac{2}{9}\right]\right), & P_{5}=\mathrm{cl}\left(\bigcup_{\text {odd }} A_{n} \cap\left[\frac{1}{9}, \frac{4}{9}\right]\right) .
\end{array}
$$

This is Markov partition because

$$
\begin{aligned}
f\left(P_{0}\right)=P_{1} \cup P_{2} \cup P_{3}, & f\left(P_{1}\right)=P_{4} \cup P_{0}, & f\left(P_{2}\right)=P_{5} \cup P_{0}, \\
f\left(P_{3}\right)=P_{2} \cup P_{4}, & f\left(P_{4}\right)=P_{3} \cup P_{5}, & f\left(P_{5}\right)=P_{0} .
\end{aligned}
$$

We can compute the intervals of the $P_{j}$,

$$
\begin{array}{crr}
I\left(P_{0}\right)=[2 / 9,8 / 15], & I\left(P_{1}\right)=[4 / 9,23 / 30], & I\left(P_{2}\right)=[5 / 9,53 / 60], \\
I\left(P_{3}\right)=[7 / 9,1 / 15], & I\left(P_{4}\right)=[8 / 9,2 / 15], & I\left(P_{5}\right)=[1 / 9,4 / 15] .
\end{array}
$$

The extreme points are interleaved and by Theorem $4.6, \mathscr{P}$ cannot factor through any connected partition.

\section{REFERENCES}

[AGW] R. Adler, W. Goodwyn, and B. Weiss, Equivalence of topological Markov shifts, Israel J. Math. 27 (1977), 49-63.

[AW] R. Adler and B. Weiss, Similarity of automorphisms of the torus, Mem. Amer. Math. Soc., no. $98,1965$.

[AM] R. Adler and B. Marcus, Topological entropy and the equivalence of dynamical systems, Mem. Amer. Math. Soc., no. 219, 1979.

[Bo1] R. Bowen, Equilibrium states and the ergodic theory of Anosov diffeomorphisms, Lecture Notes in Math., vol. 470, Springer-Verlag, 1975.

[Bo2] — On Axiom A diffeomorphisms, CBMS Regional Conf. Ser. in Math., no. 35, Amer. Math. Soc., Providence, R.I., 1978.

[Bo3] _-, Markov partitions are not smooth, Proc. Amer. Math. Soc. 71 (1978), 130-132.

[CP] E. Coven and M. Paul, Endomorphisms of irreducible subshifts of finite type, Math. Systems Theory 8 (1974), 167-175.

[CR] E. Coven and W. Reddy, Positively expansive maps of compact manifolds.

[F] D. Fried, Finitely presented dynamical systems, Ergodic Theory Dynamical Systems 7 (1987), 489-507.

[H] G. A. Hedlund, Endomorphisms and automorphisms of the shift dynamical system, Math. Systems Theory 3 (1969), 320-375.

[Kr1] W. Krieger, On sofic systems. I, Israel J. Math. 48 (1984), 305-330.

[Kr2] _ On the subsystems of topological Markov chains, Ergodic Theory Dynamical Systems 2 (1982), 195-202.

[M] B. Marcus, Factors and extensions of full shifts, Monatsh. Math. 88 (1979), 239-247.

[S] M. Shub, Global stability of dynamical systems, Springer-Verlag, 1987. 
[St] M. Stafford, Markov partitions for expanding maps of the circle, Trans. Amer. Math. Soc. 324 (1991), 385-403.

[W] B. Weiss, Subshifts of finite type and sofic systems, Monatsh. Math. 77 (1973), 462-478.

ibM Research Division, Almaden Research Center, San Jose, California 95120-6099

ibM Research Division, T. J. Watson Research Center, Yorktown Heights, New York 10598

Department of Mathematics, University of Texas at Austin, Austin, Texas 78713 